(Aus dem thierphysiologischen Laboratorium der König1. Landwirthschaftlichen Hochschule zu Berlin. Director Prof. Zuntz.)

\title{
Experimente zur Frage nach den Ursachen des Geburtseintrittes.
}

\author{
Von \\ Privatdocent Dr. Ludwig Blumreieh, \\ Assistenzarzt der geburtshïlflich-gynäkologischen Univexsitäts-Poliklinik der Königl. Charite.
}

Von jeher ist das Studium der Momente, welehe den Eintritt der zur Ausstossung der menschlichen Leibesfrucht führenden Wehenthätigkeit hervorbringen, von hohem Reiz für den Forscher gewesen. In zahlreichen vorzüglichen theils experimentellen, theils klinischen, theils anatomischen Arbeiten, sowie rein theoretischen Betrachtungen wurde versucht, das Dunkel zu lichten, in das die Ursache des Eintritts der Geburt noch gehüllt ist. Wenngleich ich in den mitzutheilenden Experimenten keineswegs die anspruchsvolle Absicht verfolge, das Räthsel der Veranlassung des Weheneintrittes zu lösen, so sind doch bestimmte Erwägungen, welche sich an einige der oben genannten Arbeiten anschlossen, der Ausgangspunkt. für meine Untersuchungen gewesen. Es sei mir daher gestattet, bevor ich auf eigene Experimente eingehe, kurz die wesentlichsten Theorien anzuführen, welche über die Ursache des Geburtsbeginns. aufgestellt sind, sowie ihre experimentelle bezw. anatomische, klische oder theoretische Begründung zu betrachten.

\section{Theorien über die Ursachen des Eintretens der Geburt.}

Mauriceau (1668) war, abgesehen von Galenus, der erste, der von der alten Ansicht des Hippocrates abwich, dass das Kind, von Hunger getrieben, sich selbst gebäre,. Er sah die Ursache 
136 Blumreich, Experimente z. Trage nach d. Ursachen d. Geburtseintrittes.

der Geburt in einer übermässigen Ausdehnung des Uterus; mit Ueberschreitung einer gewissen Dehnungsgrenze der Gebärmutter sei das Wehen auslösende Moment gegeben. Wie Veit richtig ausführt, kann indessen von einer solchen Ausdehnung des Uterus, dass derselbe gespannt ist, niemals die Rede sein, ausser "in sehr seltenen Fällen von Hydramnion, sowie ron schnell wachsenden Blasenmolen, wo es zur wirklichen Uterusspannung kommt".

Nach andern (Petit [1766], Dubois [1833], Kilian [1847]) liegt die Triebkraft zur Wehenthätigkeit in dem Druck des vorliegenden Kindstheils auf die unteren Partien der Gebärmutter. Dass in einer grossen Reihe von Fällen, wenigstens bei Erstgebärenden, ein derartiger Druck statt hat, ist zweifellos. Für zahlreiche andere Fälle aber ist die Theorie unzutreffend, denn z. B. bei Querlagen kann ein derartiges Moment nicht in Frage kommen.

Lebhafte Discussion hat die Anschaung Alexander Keilmann's hervorgerufen. Gestützt auf vergleichend-anatomische Untersuchungen von ihm selbst und seinem Schüler Knüpfer hat er folgende Hauptsätze angeführt:

1. Die für die Auslösung der Uteruscontractionen wichtigsten nervösen Apparate sind im unteren Theil der Pars supravaginalis localisirt.

2. Das Ende der Schwangerschalt ist erreicht, wenn die physiologische Entfaltung der Cervix bis zu dem in der Höhe der Seheiden-Insertion befindlichen grossen Ganglion, bezw. bis zum Ganglion cervicale Frankenhäuser's vorgeschritten ist, indem dann durch mechanische Reizung dieser Ganglien diejenigen Wehen ausgelöst werden, welche die Entleerung des Uterus zur Folge haben.

So bestechend die Lehre Keilmann's klingt und so sorgfältig auch seine und Knüpfer's anatomische Studien sind, so muss sie doch auch nur als blosse Hypothese angesehen werden. Dass sie nicht für alle Fälle zutreffen kann, geht ohne Weiteres aus der sichergestellten Thatsache hervor, dass bei der ExtrauterinSchwangerschaft, $d$. h. also bei völlig leerem Uterus, falls die Eientwickelung bis zum Ende des 10. Monats ungestört fortschreitet, sich zu diesem Termin prompt Wehen einstellen. Dieselbe Erwägung dient auch weiterhin zur Wideriegung der Allgemeingültigkeit der Lehre Mauriceau's und Kilian's.

Eine Zeit lang galt die Anschauung (Nägele 1812), dass gegen Ende der Schwangerschaft die Eihäute sich allmählich aus ihrer Verbindung mit dem Uterus loslösten, gewissermaassen ab- 
welkten, und dass nun die gelösten Eihäute als Fremdkörper contractionserregend wirkten. Aehnlich ist die Theorie von Simpson, die Decidua verfette allmählich, dadurch würde der Foetus zu einem corpus alienum im Uterus und müsse als soleher naturgemäss Wehen erregen. Die fettige Degeneration in der Decidua wurde von Kundrat und Engelmann bestätigt, dagegen als inconstant angegeben von Leopold, Langhans, Dohrn und völlig in Abrede gestellt von Winkler, Godet und Geyl. Damit ist der Theorie wohl die anatomische Basis genommen.

Eine Reihe weiterer Arbeiten wendet sich mehr chemischen Reizen zu. Nachdem Friedländer festgestellt hatte, dass gegen Ende der Gravidität zahlreiche Venenthrombosen in der Decidua serotina sich fänden, erweiterte Leopold unter Bestätigung der Angaben des Autors diesen Befund dahin, dass auch ein Theil der der Serotina zunächst gelegenen Venen der Muscularis an diesen thrombotischen Processen theilnähme und dadurch für den Blutstrom unwegsam würde. Unter Berufung auf die Ansicht BrownSéquard's, der in dem Vorhandensein von Kohlensäure im mütterlichen Blut ein mächtiges Reizmittel zur Erregung von Contractionen erblickte, betonte Leopold, dass man - die Richtigkeit der Angaben Brown-Séquard's vorausgesetzt — in der durch diese Thrombosen erzeugten venösen Hyperämie in der Placenta ein Moment von der grössten Bedeutung für die Aufklärung der ersten Wehen gefunden habe.

Gegen diese Deutung des anatomischen Bofundes scheint mir indessen einzuwenden zu sein, dass die festgesteliten Venenthrombosen ja nur geringgradiger Natur sein können; denn würden sie irgendwie erheblichere Ausdehnung erlangen und bedeutungsvoll sein, so müsste in erster Linie die Ernährung und das Leben des Foetus beeinträchtigt werden. Dies ist aber nicht der Fall. Wie schon Dohrn in seiner Kritik der Arbeit Friedländer's hervorhebt, bezieht der Foetus vielmehr gegen Ende der Schwangerschaft eine absolut grössere Menge Nährmaterials aus der Placenta als in den früheren Monaten. Und was die Versuche Brown-Séquard's anbelangt, so sind diese keineswegs beweiskräftig (genaue Besprechung der Experimente von Br.-S. findet sich in dem $\mathrm{Ab}$ schnitt: Versuche mit Kohlensäureüberladung des Blutes).

In etwas anderer Weise zieht Hasse die venöse Hyperämie als geburtseinleitenden Factor heran. Nach ihm entwickelt sich gegen Ende der Gravidität ein stärkerer Kohlensäurereichthum in 
138 Blumreich, Experimente z. Frage nach d. Ursachen d. Geburtseintrittes.

der foetalen Aorta und damit auch in den Nabelarterien. Die allmählich eintretende vollkommene Ablenkung des Blutstromes der Vena cava inferior von der linken Vorkammer nach der rechten Kammer hin, ferner die relative Kaliberabnahme des Ductus venosus Arantii und des Ductus Botalli bringt eine derartige erhöhte Venösität zu Stande. „Der rechtzeitige Eintritt der Geburt ist alsdann abhängig von der Einwirkung eines bestimmten Gehaltes des in die fötale Placenta strömenden Blutes an Kohlensäure auf die nervösen Centralapparate der Musculatur des Uterus."

Dieser Auffassung Hasse's gegenüber möchte ich bemerken, dass das Blut der Nabelarterien, nachdem es in die Placentarsinus gelangt ist, von diesen aus mit der Museulatur und den Nerven des Uterus überhaupt nicht in Berübrung kommt, sondern direct in die Uterusvenen abgeführt wird. Es gelangt also erst nach Passage des Herzens und der Lunge wieder zur Gebärmutter zurück, ist aber natürlich auf diesem Wege schon wieder von der Koblensäure befreit worden. Um also eine Beeinflussung der Uterusmusculatur durch den Gasgehalt des Placentarblutes anzunehmen, müsste man geradezu die Hypothese aufstellen, dass besondere sensible Nerven von dem Placentargebiet zum Nervensystem führten, welche den durch das kohlensäurereichere Blut in der Placenta materna gesetzten Reiz auf die Gebärmuttermusculatur selbst übertrügen - eine Annahme, der jede anatomische Grundlage fehlt.

Hasse hält indessen noch andere Stoffe des fötalen Blutes für bedeutungsvoll; vielleicht sei es nicht allein die Aenderung des Gasgehaltes, sondern auch irgend welche sonstigen Körper der regressiven Metamorphose im Fötalblut, die hier in Betracht kommen. Der letzteren Anschauung neigte sich Liedke zu und empfahl experimentelle Untersuchungen betreffend den Einfluss des Harnstoffgehaltes im Blut auf die Bewegungen des Uterus anzustellen. Auf dem Boden dieser Hypothese Hasse's und Liedke's prüfte Feis das Verhalten des Uterus bei pathologiseher Harnstoffanhäufung im Blut. Diese Experimente fielen indessen negativ aus; die Gebärmutter wurde nicht zur Contraction gebracht.

Runge stellte dann sehr interessante Experimente am nichttragenden Thier an. In einer Reihe von Fällen wurde das Blut mit Kohlensäure überladen, in einer anderen Serie künstlich Saverstoffmangel erzeugt. Er kam nach seinen Ergebnissen zu dem Schluss, dass beide Momente im Stande seien, Contractionen aus- 
zulösen, dass indessen der Sauerstoffmangel das wirksamere Agens bilde.

Ich werde weiterhin noch sehr ausführlich auf die Arbeit Runge's einzugehen haben. Nur soviel sei jetzt hier bemerkt, dass man aus dem Effect bestimmter reizender Medien auf den Uterus des nichttragenden Thieres kaum sichere Schlüsse auf das Verhalten der Gebärmuttermusculatur tragender Individuen za ziehen berechtigt ist.

Weniger Anerkennung haben schliesslich einige andere Theorien gefunden, die ich noch erwähnen möchte, wie die von Ritgen, der die Ursache des Geburtseintrittes in der vollendeten Ausbildung der Muskelfasern sah, sowie die Anschanung von Litzmann: die vollkommene Ausbildung der Nervenfasern bedinge es, dass sie nun allmählich zur Wirksamkeit gelangten.

Man sieht aus dem Angeführten, dass wir bezüglich der Ursachen des Geburtseintrittes noch völlig im Dunkeln tappen. Gegen jede der Theorien lassen sich Einwände erheben, zum mindesten dagegen, dass sie für alle Fälle zutreffend sind; nalle bisher gegebenen Erklärungen für die Ursache des Geburtseintrittes sind durchaus hypothetischer Natur" (Runge).

Ich bin nun bei der Anstellung meiner Experimente von folgender Ueberlegung ausgegangen. Die Geburt setzt sich zusammen aus zwei Momenten; wir haben es zu thun a) mit einem Reiz, der auf die Musculatur des graviden Uterus cinwirkt, b) mit der Thätigkeit der durch den Reiz beeinflussten Muskelmasse des schwangeren Tragsackes.

So genau wir über Punkt b) orientirt sind, so wenig wissen wir über die in Frage kommenden Reizeinflüsse. Hier ist nicht nur der endgültige Reiz, der die Wehenthätigkeit auslöst, unbekannt, sondern wir wissen überhaupt sehr wenig über das Verhalten des graviden Uterus im Vergleich mit der nicht schwangeren Gebärmutter Reizen gegenüber. Ziemlich allgemeine Verbreitung besitzt die Anschaung, dass der Uterus im weiteren Verlaufe der Schwangerschaft eine erhöhte Reizempfindlichkeit besässe. „Je weiter die Gravidität fortgeschritten ist, desto geringere Reize genügen, um wirklich Wehen herbeizuführen" (Veit). Es schien mir sehr fraglich zu sein, ob diese Lehre in dieser Ausdehnung zutreffend ist. Die glatte Muskelfaser wird durch verschiedene Reizqualitäten in ganz verschiedener Weise beeinflusst. Die eine Reizart erregt kaum eine sicht- und messbare Verkürzung, die andere führt 
140 Blamreich, Experimente z. Fraga nach d. Lrsaehen d. Geburtseintrittes.

zur lebhaftesten Contraction. Ganz ebenso verhält sich ja auch der Complex glatter Muskelfasern, den man als Uterus bezeichnet; er reagirt keineswegs auf alle Reize in genau derselben Weise. Und nun erst die Gebärmutter in der Gravidität! Wir haben es ja hier mit einem Organ von ganz besonderer Eigentbümlichkeit zu thun, eivem Gebilde, das während der ganzen Dauer der Schwangerschaft in ständiger Weiterentwickelung, Veränderung begriffen ist. Die Entwickelungs- und Rückbildungsvorgänge, die der gravide Uterus durchmacht, sind von solcher Eigenart, dass wir uns vergebens nach einem Analogon in der Biologie umschauen. Ls wäre durchaus möglich, dass die Gravidität, die sehr zahlreiche ejgenthümliche Veränderungen des Körpers mit sich führt, die sicher den Chemismus in dieser Zeit sehr erheblich beeinflusst, durch irgend welche Momente - vielleicht chemische - oder auch durch Cireulationsabnormitäten die Erregbarkeitsverhältnisse der Uternsmusculatur wesentlich beeinflusst, so dass Reize, die auf den nicht schwangeren Uterus energisch einzuwirken vermögen, beim tragenden Organ versagen und umgekehrt andere wieder entweder nur oder in viel höherem Maasse an der graviden Gebärmutter ihre Wirksamkeit entfalten können. Die Ergebnisse von Reizversuchen an dem ruhenden inactiven Organ, dass die nicht schwangere Gebärmutter darstellt, berechtigen uns daher meines Lrachtens noch nicht zur Uebertragung derselben auf dasselbe Gebilde im graviden Zustande. Und hier muss, wie mir scheint, die Analyse einsetzen.

Mein Versuchsplan war daher festzustellen:

1. wie verbält sich der Uterus des normalen nichttragenden Thieres gegenüber verschiedenen Reizqualitäten,

2. wie verhält sich der gravide Fruchthalter derselben Thierspecies gegenüber diesen Reizen, speciell gegenüber solchen, die in den Theorien über die Ursache des Geburtseintritts eine Rolle spielen. Nimmt die Reizbarkeit für alle Reizarten gleichmässig zu? Oder giebt es gewisse Reize, die besonders energisch auf die Musculatur des graviden Uterus einwirken? Giebt es also Reize, die gewissermaassen den "adäquaten" Reiz für den schwangeren Tragsack darstellen? Oder handelt es sich nur um eine allgemein erhöhte Irritabilität, die allen Reizeinflüssen gegenüber in derselben Weise verstärkt ist? Ich durfte hoffen, aus der experimentellen Beantwortung dieser Fragen einen bescheidenen Beitrag zur Jüsung der wichtigsten Vorfrage für das Studium der Ursachen des Ge- 
burtsbeginns, der Reizempfindlichkeit des graviden Uterus, vielleicht auch einige weitere Ausblicke zu gewinnen.

Bevor ich auf eigene Experimente eingehe, möchte ich in der nachfolgenden tabellarisch gehaltenen Uebersicht angeben, was in Bezug auf die Reizung des Uterus im Wesentlichen bisher gearbeitet worden ist, namentlich, ob dabei Verschiedenheiten zwischen dem Verhalten der graviden und leeren Gebärmutter sich ergeben haben. Ich kann mich im Uebrigen hier um so kürzer fassen, als die meisten Untersuchungen in der Absicht angestellt worden sind, die Innervations-Verhältnisse der Gebärmutter zu ergründen, ein Punkt, der bei meiner Fragestellung gänzlich ausser Acht gelassen wurde. Das wissenschaftlich berechtigste Eintheilungs-Princip wäre vielleicht das nach dem Angriffspunkt des Reizmittels; man könnte also eine Eintheilung vornehmen, je nachdem erstens die nervösen Centralorgane des Uterus oder zweitens "die Gebärmutter direct oder drittens reflectorisch auf den Uterus wirkende Nerven bez. Körper-Regionen gereizt würden.

Indessen wärde diese Eintheilung auf gewisse Schwierigkeiten und Unzweckmässigkeiten stossen, einmal weil bei manchen Reizqualitäten, wie beispielsweise bei den Veränderungen des Gasgehaltes des Blutes, keine Einigkeit darüber besteht, ob wir es hier mit einer äirecten Erregung der Gebärmutter-Substanz oder mit einer Reizung der nervösen Centren zu thun haben, ja ob nicht auch reflectorische Einflüsse mitspielen und weil ich ferner manche Reizart bei jeder einzelnen der 3 Unterabtheilungen besprechen müsste. Es scheint mir daher richtiger zu sein, eine Eintheilung nach den Reizqualitäton vorzunehmen.

\section{Uebersicht über die bisherigen Reizversuche am Uterus.}

\section{Elektrische Reize.}

a) Directe Reizung. Während von Basch und Hoffmann feststellten, dass der Erfolg der directen Reizung des leeren und des tragenden Hundeuterus ein sehr inconstanter ist und der Effekt häufig ausbleibt, theilt Onimus mit, dass die Hundegebärmutter in nichttragendem Zustande allerdings wenig zu beeinflussen sei, dass dagegen die Gebärmutter des schwangeren Thieres, wenn erst einmal die Wehen aus irgend einem Grunde angefangen haben, sehr energisch durch den elektrischen Strom erregt wird. Die Resultate am Kaninchen-Uterus sind nicht ganz die gleichen. Kehrer reizte 
142 BIumreich, Experimente z. Frage nach d. Ursachen d. Geburtseintrittes.

den Kaninchen-Uterus durch den galvanischen Strom mit positivem Erfolg. Nach Körner ist die directe Reizung der Gebärmutter ebenfalls erfolgreich, und zwar dauern die Bewegungen um so länger an, ein je stärkerer Reiz das Organ trifft. Jakub fand, dass intermittirende elektrische Reizung die vorhandenen Zusammenziehungen verstärkt und neue hervorruft. Auch Röhrig fand Contractionen nach directer clektrischer Reizung. Die vier Autoren geben nicht an, ob Unterschiede in der Stärke der Reaction zwischen graviden und nichtgraviden Thieren vorhanden waren. Auch Bumm schreibt nur, dass bei directer Reizung mittelst galvanischen und faradischen Stromes bei trächtigen und nichtträchtigen Thieren $\mathrm{Zu}$ sammenziehungen erzielt wurden. - Cohnstein fand gewisse Unterschiede zwischen trächtigen und nichtträchtigen Kaninchen, soweit das aus seinen fast aphoristisch gehaltenen Mittheilungen zu ersehen ist: Directe Gebärmutter-Reizang durch den inducirten Strom erzeuge bei trächtigem Uterus intensive Contraction, wenn die hintere Vaginalwand, bei nichtträchtigem Uterus, wenn die Hörner berührt werden.

b) Reizung der nervösen Centren bezw. der UterusNerven. Spiegelberg, Körner, Obernicr und Röhrig stellten übercinstimmend fest, dass von den versehiedensten Stellen des Rückenmarks aus sich Contractionen auslösen lassen, dass dieselben aber um so stärker werden, je mehr die Einstichstelle zur Medulla lumbalis heranrückt. Auch Jakub fand die Reizung des Lendenmarks am wirksamsten. Die Reizung des Cerebellum, sowie verschiedener Gehimpartieen ist nach Obernier, Körner und Röhrig auch wirksam, aber in schwächerem Maasse. Nach Kehrer löst Reizung der Rami sacr. eines Plexus hypogastr. post. Contractionen in den nach dem Tode ruhenden Genitalien aus, Reizung des Pl. hypogastr. magn. nicht mit Sicherheit.

Körner zeigte, dass elektrische Reizung der zum Uterus gehenden Sacraläste, ferner Reizung des Sympathicus der Bauchaorta Contractionen erzeuge; cinen sehr eklatanten Erfolg von der Reizung der Sympathicusstämme in der ganzen Lumbalgegend sowie der Nerven-Plexus, welche dio Vasa abdominalia begleiten, sah auch Obernier. Frankenhäuser hält sogar den Plexus aorticus für den eigentlichen motorischen Nerv des Uterus. Während Kilian fand, dass elektrische Reizung der Nervi vagi Uterus-Bewegungen auslöse, kamen Spiegelberg und Obernier zu dem entgegengesetzten Resultate. 
Blumreich, Experimente z. Frage nach d. Ursachen d. Geburtseintrittes. 143

Auf gewisse Unterschiede zwischen tragenden und nichttragenden Thieren machen dann von Basch und Hoffmann, Obernier, Cohnstein und Dembo aufmerksam.

Von Basch und Hoffmann farden, dass elektrische Reizung der N. hypogastr. Contractionen der Ringmuskelfasern des Uterus der Hündin hervorruft, besonders bei tragenden Thieren soll elektrische Reizung der $\mathrm{N}$. erigentes Contractionen der Längsmuskelfasern erzeugen. Obernier stellte fest, dass bei 3 im Anfang der Schwangerschaft befindlichen Kaninchen nach schwacher sowie starker Rückenmarksreizung Uterus-Bewegungen ganz ausblieben. Auch Reizung des Bauch-Sympathicus erzeugte nur in einem Fall schwache Uterus-Bewegungen. Wie viel von den übrigen 42 Kaninchen schwanger waren und mit welchem Erfolg sie gereizt wurden, lässt sich aus seiner Arbeit nicht ersehen; er schliesst aus seinen Experimenten auf eine herabgesetzte Erregbarkeit im Beginn der Schwangerschaft. Eine Bestätigung findet das durch Cohnstein. O. macht darauf aufmerksam, dass elektrische Reizung des Lendenmarks bei trächtigen Thieren Contractionen gewöhnlich weder auslöst, noch bereits vorhandene verstärkt, dass dagegen bei nichtträchtigen Thieren Zusammenziehungen darnach eintreten. Auch Dembo berichtet nach seinen, wie es scheint, allerdings sehr spärlichen Versuchen, dass auf elektrische Reizung von den verschiedensten Stellen aus der gravide Uterus sich viel weniger erregbar zejge, als der nichtschwangere. Geburt oder Frühgeburt herbeizuführen gelang nicht.

c) Auf reflectorische Reizung verschiedener peripherer Nerven des Stammes veranlasst auch Schlesinger den Uterus zu den kräftigsten Bewegungen. Ja nach Röhrig gebört sogar die elektrische Reizung einer Reihe centraler Nervenstümpfe zu den allerwirksamsten Hülfsmitteln zur Auslösung kräftiger Uterusbewegungen. Wie wirksam die reflectorische Reizung ist, lehrt auch die Beobachtung von Basch und Hoffmann, welche angeben, dass die Reizung des Ischiadieus ungleich wirkungsvoller bezüglich der Erzeugung der Uteruscontractionen beim Hunde ist, als die AthmungsSuspension.

Ueber die in diesen Arbeiten erörterte Frage nach dem Einflusse des Centralnervensystem sauf den Uterus und nach den Nervenbahnen, welche diese Einflüsse vermitteln, sind Untersuchungen von mir nicht angestellt worden und ich enthalte mich deshalb einer näheren Kritik derselben. Was in einiger Beziehung za meiner Fragestellung steht, ist, dass mehrere dieser Untersuchungen eben- 
144 Blumreich, Experimente z. Frage nach d. Ursachen d. Geburtseintrittes.

falls die Erregbarkeit des Utorus in den verschiedenen Lebensphasen und zwar durch elektrische Reize erörtern. Nach dem Ergebniss dieser Arbeiten kann man es wobl als festgestellt betrachten, dass gewisse Unterschiede bestehen und zwar in der Art, dass die gleiche Reizqualität - hier die elektrische Reizung - in den verschiedenen Lebenszeiten verschiedene Effekte auslöst.

\section{Thermische Reize.}

Als erster hat Calliburcès thermische Reize aul den Uterus einwirken lassen. Er fand, dass sowohl der trächtige, als auch der nichttragende Uterus von Hunden, Ratten und Kaninchen auf trockene und feuchte Wärme durch energische Zusammenziehungen reagire. Auch Kehrer erkannte unter Berufung auf Calliburces in der Wärmeentziehung und -Zufuhr ein Mittel, um rythmische Bewegungen in den bis dahin ruhenden Genitalien zu erwecken. Beim Hund hat nach von Basch und Hoffmann der thermische Reiz den constantesten Erfolg. Auch Cohnstein hält heisses Wasser für eines der kräftigsten Reizmittel für den leeren und trächtigen Uterus. Röhrig und Dembo, ferner Reimann, welch' letzterer am herausgeschnittenen Organ experimentirte, bestätigen den contractionserrcgenden Einfluss dieser Reizqualität. Reimann macht indessen darauf aufmerksam, dass eine starke Erhöhung oder Erniedrigung der Temperatur die Bewegungen der Gebärmutter augenblicklich unterdrücke. Diese Beobachtungen wurden weiterhin bestätigt von Runge und Frommel. Runge's Experimente am jungfräulichen geschlechtsreifen Kaninchen ergaben, dass je höher die Temperatur des in die Bauchhöhle eingelassenen heissen Wassers wird, desto lebhafter die Contractionen sind, aber anch desto kürzer ihre Dauer, desto intensiver das nachfolgende Lähmungsstadium. Bei sehr hoher Temperatur ist sogar der sofortige Tod des Uterusgewebes die Folge. Auch heisse Luft mittelst Thermokauter angewendet, erwies sich als starkes Reizmittel. Eiswasser machte sofort starke tetanische Zusammenziehungren der Gebärmutter. Ebenso wie heisses Wasser wirkte erhöhte Körpertemperatur als contractionserregendes Nittel. Frommel wies dann schliesslich darau hin, dass bedeutende Abnahme der Körperwärme die Contractionen verlangsame, Steigerung der Blutwärme zunächst die Zusammenziehungen beschleunige, dass indessen hohe Fiebertemperatur Uterusbewegungen völlig aufhcbe. 
B lumreich, Experimente z. Frage nach d. Ursachen d. Geburtseintrittes. 145

\section{Mechanische Reize.}

Directe Reizung der Gebärmutter durch Berührung oder Streichen der Aussen- und Innenfläche, ferner Einspritzung von Flüssigkeit in die Genitalhöhle, wirkt nach Kehrer contractionsauslösend. Dagegen erregt nach ihm mechanische Reizung des Plexus hypogastricus magnus nicht mit Sicherheit Bewegungen in den ruhenden Genitalien. Nach Körner ist die direete mechanische Reizung um so erfolgreicher, je stärker der Reiz ist, der das Organ trifft. Auch Röhrig und schliesslich Jakub bestätigen den contractionserregenden Effect der directen mechanischen Einwirkung. Saugen an den Brustwarzen, überhaupt mechanische Reizung derselben erzeugt ebenfalls reflectorisch mehr oder weniger lebhafte Contraction der Gebärmutter. So wirkt reflectorisch auch nach Röhrig Kitzeln des Scheideneinganges mit einer Federspule.

Die Angaben über die mechanische Erregung des Vterus sind indessen bei allen Autoren ausserordentlich kurz gehalten.

4. Störung in der Blut-Circulation und im Gasgehalt des Blutes.

Die Reizmittel, mit denen hier gearbeitet wurde, waren Athmungs-Suspension, ferner Compression der Aorta, Tödtung durch Verblutung, Injection von Blut in die Bauchaorta, schliesslich künstlich erzeugte rasche Kohlensäureüberladung des Blutes und artificieller Sauerstoffmangel im Gefässsystem.

Die Literatur über diese Reizarten muss indessen ausführlich besprochen werden und verweise ich daher auf die Abschnitte: Versuche mit Kohlensäureüberladung des Blutes und Versuche mit Saucrstoffmangel des Blutes.

\section{Ohemische Reize.}

Kehrer fand, dass Säuren und Alkohol Bewegungen des Uterus durch directe chemische Reizungen des Uterus hervorriefen; dagegen löste die chemische Reizung des Pl. hypogastr. magn. Contractionen nicht mit Sicherheit aus. Auch Körner bestätigt den Einfluss der directen chemischen Errogung des Uterus, ohne sich indessen näher darüber auszulassen.

Röhrig injicirte eine grosse Reihe chemisch toxischer Substanzen und fand starke contractionsauslösende Wirkung, namentlich beim Strychnin, ferner besonders bei Ammoniak, Picrotoxin, Ergotin, Calabar, Aloe, Coloquinten und Sabina. Bei der directen Reizung 
146 Blumreich, Experimente z. Frage nach d. Ursachen d, Geburtseintrittes.

erwies sich Essigsäure und Ammoniak-Lösung als besonders wirksam. Die contractionssteigernde Wirkung des Strychnins betonte auch Jakub.

Von den vielen Arbeiten, welche über Sekale und seine Derivate in ihrer Beziehung zu den Uterus-Contractionen angestellt sind, will ich nur auf die neueren Arbeiten von Nikjtin und von Markwald verweisen. Ergotinum dialysatum und Sklerotin-Säure machte bei trächtigen, jungfräulichen und puerperalen Thieren kräftige Zusammenziehungen des Uterus, die aber rasch vorübergingen und sich nicht zum Tetanus steigerten. Ergotinin dagegen war erfolglos. Schliesslich hat Feis Harnstoff in die Blutbahn gebracht und zwar in solehen Mengen, dass es zu starken allgemeinen Krämpfen und sogar zum Exitus des Thieres kam, ohne dass indessen gleichzeitig Contractionen der Gebärmutter ausgelöst wurden.

\section{Methodik.}

Da meine Versuche nicht bezwecken, die Reizbarkeit des Uterus überhaupt zu prüfen, sondern vielmehr relativ feine Unterschiede in dem Verhalten der Empfindlichkeit der Gebärmutter verschiedenen Reizen gegenüber in nicht gravidem und gravidem Zustande festzustellen, musste eine Thierspecies zur Verwendung gelangen, deren Uterus verhältnissmässig geringer Reize bedarl, um zur Contraction zu kommen. Denn je intensivere Reizeinwirkungen von Nöthen sind zur Auslösung irgend eines Effectes, desto schwerer lassen sich Normen für Unterschiede in der Reizempfänglichkeit aufstellen. Aus dem Studium der Literatur und eigenen Versuchen überzeugte ich mich, dass Meerschweinchen nicht sehr geeignet sind wegen der Kleinheit der Gebärmutter, wodurch die Beobachtung von Zusammenziehungen überhaupt sehr erschwert ist. Katzen und Hunde sind andererseits nicht zweckmässig, weil hier das nothwendige Moment der leichten Erregbarkeit gänzlich fehlt. So blieb denn von den üblichen Versuchsthieren nur das Kaninchen übrig. Aber auch hier sind nicht unerhebliche Schwierigkeiten zu äberwinden. Die Reizempfindlichkeit der Gebärmutter dieser Thiere schwankt in ausserordentlich weiten Grenzen, und nur die sorgfältigste Beachtung der physiologischen Unterschiede in der Irritabilität vermag hier vor dem Irrthum zu schützen, Contractionsphänomene, die einfach auf gewissen physiologisehen Verhältnissen beruhen, als Reizeffeete anzusprechen - eine Thatsache, die auch andere Experimentatoren mit Recht nachdrücklich betont haben. 
Am störendsten werden vor allen Dingen die sogenannten Spontancontractionen des Uterus empfunden. Eröffnet man nämlich die Bauchhöhle eines schwangeren Kaninchens, so sieht man, namentlich wenn das Thier sich gegen Ende der Gravidität befindet, wie sich mehr oder weniger lebhafte Zusammenziehungen über kleinere und grössere Abschnitte des Uterus fortpflanzen. Die Bewegungen sind ziemlich geordnet, tragen einen ausgesprochen peristaltischen Charakter und scheinen ohne jeden äusseren Einfluss aufzutreten. Ich kann Röhrig ganz Recht geben, wenn er sagt, dass solche Contractionsphänomene am schwangeren Organ oft Stunden lang andauern, doch möchte ich einschränkend betonen, dass erstens diese sehr lange anhaltenden Spontancontractionen sich nur am Uterus gravidus in der letzten Woche finden, dass dagegen in den ersten drei Schwangerschaftswochen die Dauer und Intensität derselben wesentlich geringer ist, und zweitens, dass auch hier recht erhebliche Unterschiede vorhanden sind. Jch habe gravide Uteri gesehen, bei denen sich alsbald nach Eröffnung der Bauchhöhle eine äusserst ausgesprochene Peristaltik präsentirte, und wieder andere, ebenfalls am Ende der Schwangerschaft, bei denen die Zusammenziehungen nur geringgradig waren und nach kurzer Zeit, $1 / 4-1 / 2$ Stunde völlig nachliessen. Das andere Extrem bildet der Uterus des jungfräulichen Kaninchens, der nach der Ansicht der meisten Experimentatoren keine Spontancontractionen aufweist. Wenig brauchbar zu Reizversuchen ist der Uterus dieser Thiere, solange das Alter der Geschlechtsreife nicht erreicht ist, also etwa in den ersten 5-6 Monaten. Die Gebärmutter ist hier fadenförmig, sehr wenig Musculatur enthaltend, fast noch fibrös. Gegenüber Frommel muss ich mit anderen Autoren, wie Sigmund Mayer, Palm u. s. w., nach meinen Versuchen sagen, dass der Uterus dieser ganz jungen Thiere recht schlecht auf relative energische Reize reagirt. Eine reichlichere Entwickelung der Musculatur setzt erst mit der Heranreifung zur Geschlechtsthätigkeit ein; der Uterus wird bandförmig, das gelbliche Colorit, das sofort das unentwickelte Organ verräth, verwandelt sich in rosenrothe Farbe. Namentlich Oser und Schlesinger haben den Uterus des jungfräulichen, aber geschlechtsreifen Thieres zu Reizversuchen empfohlen, weil die Reizempfindlichkeit in dieser Zeit eine genügend gute ist und andererseits Spontancontractionen gänzlich fehlen.

Zwischen diesen beiden Extremen des hochschwangeren und 
148 Blumreich, Experimente z. Frage nach d. Trsachen d. Geburtseintrittes.

des jungfräulichen Uterus steht die Gebärmutter älterer Thiere, die kurz nach dem Werfen sind nder die überhaupt schon ein oder auch mehrere Male geboren haben. Hier sind Spontancontractionen wahrnehmbar, aber man kann, wie Röhrig das auch hervorhebt, darauf rechnen, dass diese Zusammenziehungen nach einiger Zeit gänzlich nachlassen. Röhrig giebt 3 bis 10 linuten an. Ich sah die Bewegungen manchmal bis za 14 Vinuten anhalten, ehe völlige Ruhe eintrat.

Ich möchte indess hervorheben, dass gerade bei dieser Gruppe der Thiere die individuellen Sehwankungen am grössten sind. Neben vereinzelten vollreifen, geboren habenden Kaninchen, die keinerlei Spontancontractionen aufweisen, stehen andere, bei denen die Intensität derselben sehr gering ist und ein deutliches Nachlassen der Zusammenziehungen nach 2 bis 3 Minuten eintritt, und wieder andere, bei denen die Lebhaftigkeit der Spontanbewegungen an die beim hochschwangeren Thier erinnert und bei denen manehmal fast eine halbe Stunde vergeht, ehe das gänzliche Aufhören der Contractionen erfolgt und damit das Thier zum Reizversuch geeignet ist.

Dieser Ansicht gegenüber, zu der zahlreiche eigene Beobachtungen und die Beschäftigung mit der Literatur geführt haben, steht die Ansehauung von Frommel, dass der Kaninehen-Uterus zo jeder Zeit, also auch im infantilen und virginellen Stadium rythmische Spontancontractionen ausführt. Bei der Wichtigkeit seiner Versuchsanordnung und Ergebnisse sei es mir gestattet, in einiger Ausführlichkeit auf Frommel's Untersuchungen einzugehen.

Der Autor ging davon aus, dass es eine unvollkommene Methode sei, wenn man - wie es alle früheren Forscher thaten die Genitalien einfach freilegte und nun mittelst des Gesichtssinnes die Effecte der verschiedenen Reizungen constatirte. Fr liess daher den Uterus unter Anwendung der graphisehen Methode seine Bewegungen selbst an der vorübergeführten Trommel anschreiben, um so vor Selbsttäuschungen in der Beobachtung gesichert zu sein.

$\mathrm{Zu}$ diesem $Z_{\text {weck }}$ bediente er sich eines sehr ingeniösen Apparates. Nachdem die Gebärmutter durch die Banchwonde hervorgezogen war, wurde eine Incision in die Scheide gemacht und hier ein Glasröhre so weit eingeführt, bis sie die beiden Muttermunde erreichte, und nun durch Fäden fixirt. Mit der Glasröhre in Verbindung stehen $z$ wei Rohre, deren eines direct in ein Manometer überging, während durch das andre körperwarme $1 / 2$ pro- 
centige Kochsalzlösung zugeführt wurde. Ferner war in jedem der beiden Uterushörner eine kleine Glascanüle eingebunden; diese beiden Canülen wurden dann durch eine gabel(örmige Glasröhre vereinigt, an deren Stieltheil ein Gummischlauch zwecks Abklemmens angebracht war.

Nunmehr wurde der Uterus dureh die auf Körperwärme gehaltene Kochsalzlösung aufgespritzt; sobald die Flüssigkeit aus dem Gummischlauch hinter den Uterushörnern ausfloss, worde derselbe abgeklemmt und der Hahn an der Zuleitungsröhre geschlossen. Durch Oeffnen des Hahnes am Manometerrohre steigt in diesem die. Flüssigkeit entsprechend dem intrauterinen Druck, und das Manometerrohr wird nunmehr mit der Marey'schen Luftkapsel in Verbindung gebracht, so dass jetzt jede kleinste Bewegung der Flüssiglseit im Manometerrohr durch den Hebel am Kymographion registrirt wird. Die am Kymographion aufgeschriebene Curve entspricht darn genau der während der Contraction aus dem Uterus verdrängten Flüssigkeitsmenge.

Frommel kommt zu dem Ergebniss, „dass der Uterus in allen Stadien seiner Entwickelung die Fähigkeit besitzt, rythmische Contractionen auszulösen. Und zwar ist der Rythmus bei trächtigen Kaninchen und solchen, welche bereits geboren haben, ein regelmässiger, während er beim infantilen Uterus meist weniger regelmässig angeordnet erscheint."

Gerade dieses Resultat indessen seheint mir gegen die allgemeine Brauchbarkeit der Methode zu sprechen, so vorzüglich erdacht dieselbe auch ist. Wenngleich ich zugebe, dass gelegentlich auch der jungfräuliche Uterus Spontancontractionen aufweist, so habe ich doch in der weitaus grössten Zahl meiner Vorversuche ${ }^{1}$ ) auch nicht die leiseste Andeutung solcher Bewegungen, selbst nicht bei schärfster Lupenbeobachtung constatiren können. Ich muss mich daher den früheren Autoren, besonders Oser, Schlesinger, Röhrig und Runge, anschliessen mit der Behauptung, dass der virginelle Uterus Spontancontractionen nicht aufweise. Ich glaube, dass die Ursache der rythmischen Contractionen, welche Frommel bei allen sejnen Thieren beobachtet hat, in der dauernden mechanischen Reizung liegt, die auf den Uterus durch das Eingebundensein der Canülen und die Anfüllung mit Flüssigkeit ausgeübt wird. From mel

1) Bei den eigentlichen Versuchen habe ich stets mit dem Kochsalzwasserbade gearbeitet, und hier liegen die Verhältnisse, wie weiter ausgeführt wird, etwas anders. 
150 Blumreich, Experimente z. Frage nach d. Ursachen d. Geburtseintrittes.

sucht diesen Einwand selbst zu entkräften, indem er darauf hinweist, dass die Verhältnisse analog wie beim Froschherzen liegen. Aus den Arbeiten der Ludwig'sehen Schule sei bekannt, dass das mit Canülen versehene Froschherz den feinsten Einflüssen der Reizund Ernährungsbedingungen zugänglich bleibt.

Dieser Analogieschluss scheint mir aber nicht ohne weiteres berechtigt zu sein. Das Herz wird in normalem Zustand von Flüssigkeit durchströmt, für den Uterus dagegen ist das Aufspritzen mit einem flüssigen Medium ein Reiz durchaus ungewöhnlicher Art.

Aus den Resultaten der Frommel'schen Versuche scheint mir daher nur hervorzugehen, dass bei Anwendung seiner Methode unter allen Umständen rythmische Contractionen im Kaninchenuterus ausgelöst werden, nicht aber, dass diese Bewegungen wirkliche Spontanbewegungen, d. h. unabhängig von der Methode eintretende Contractionen sind. Wie ich einem Citat in Peter Müller's Handbuch der Geburtshilfe entnehme, - die Originalarbeit war mir leider nicht zugänglich - hat Conrad die Contractionen, welche Frommel schildert, ebenfalls der Methode zur Last gelegt.

Ueber den Ursprung der Spontancontractionen sind die Ansichten der Autoren getheilt. In seinen experimentellen Untersuchungen zur Innervation der Gebärmutter schreibt Cohnstein: "Diese Contractionen sind bedingt durch die während der Schwangerschaft im Parenchym der Geschlechtsorgane stattfindenden Veränderungen und hören allmälig auf mit der puerperalen Involution." Eine Motivirung dieses Standpunktes habe ich in seinen Auslassungen nicht finden können. Kehrer meint, dass diese sogenannten Spontanbewegungen nur rythmische Nachwirkungen auf einen primären Reiz seien, welcher je nach der Irritabilität des Uterus verschieden gross sein müsse. So sei bei sehr reizbarem Organ schon der Luftzutritt nach Eröffnung der Bauchhöhle ausreichend zu ihrer Erregung, in anderen Fällen seien Störungen in der Circulation oder directe Reize des Uterus erforderlich, um eine solche Folge von Nachwirkungen auszulösen.

Das Zustandekommen sei unbedingt geknüpft an das Erhaltensein der zum Uterus führenden Krenzbeinnerven sowie an die Anwesenbeit einer gewissen Blutmenge in dem Genitalorgan.

Nach wieder Anderen, wie Kilian, sind es im Wesentlichen. äussere Reize, Einflüsse dẹ atmosphärisehen Luft, Temperatur des Versuchsraumes, zufällige mechanische Erschütterungen des Organs a. s. w., auf welche der reizbare Uterus des tragenden und des bereits 
Blumreich, Experimente z. Frage nach d. Ursachen d. Geburtseintrittes. 151 geborenhabenden Kaninchens mit länger dauernden Zusammenziehungen antwortet.

Meine Erfahrungen lehren, dass äussere Reize jedenfalls in nicht unerheblichem Grade bei der Erzeugung dieser Contractionen mitspielen. Und zwar muss man das wohl deshalb annehmen, weil bei Fernhaltung derselben durch Experimentiren im Kochsalzwasserbade eine schr wesentliche Herabsetzung der Spontancontractianen eintritt, wenigstens soweit, dass sie die Prüfung der Reizeffekte nicht mehr übermässig erschweren. Es bilden ja die Spontancontractionen des tragenden Thieres eine Klippe, an der der ganze Versuchsplan fast gescheitert wäre; denn wie soll man Reizeinwirkungen und Effekte studiren, wenn man nicht entscheiden kann, ob das beobachtete Contractionsphänomen dem angewandten Reizmittel oder der Eigenbewegung des Uterus entspricht.

Aus dieser Verlegenheit hilft indessen das Wasserbad. Setzt man vor Eröffnung der Bauchhöhle die untere Rumpfhälfte des Thieres unter physiologische Kochsalzlösung, die andauernd bei Körpertemperatur erhalten wird, so wird ausgeschaltet a) der austrocknende Einfluss der Luft, b) die Luftbewegungen, c) die directen Temperatureinflüsse der Aussenluft auf den Uterus, d) die Abkühlung des Körpers, welche dureh die Fesselung, zumal bei eröffnetcr Bauchhöhle erzeugt wird. All diese Momente sind, wie mir scheint, von sehr wesentlicher Bedeutung: Dem gegenüber steht als Nachtheil der mechanische Reiz, den'die Flüssigkeit im Augenblick des Eindringens in die Bauchhöhle ausübt. Auch wenn noch so behutsam beim Einbringen in das Wasserbad verfahren wird, bekommen die Därme einen Auftrieb und dadurch werden leichte Dislocationen und Zerrungen veranlasst. Aber die dadurch zunächst hervorgerulenen Contractionen beruhigen sich nach relativ sehr kurzer Zeit, so dass man alsdann ungestört experimentiren kann. Ish habe geradezu den Eindruck gehabt, als ob die körperwarme physiologische Kochsalzlösung einer beruhigenden Einfluss auf die durch irgend welche Reizeinflüsse bereits entstandenen Contractionen ausübt.

$\mathrm{Zu}$ meiner Freude hat $\mathrm{Palm}^{\mathrm{y}}$, erster Assistent von Runge, vor kurzem mitgetheilt, dass er bei der Nachprüfung der Versuche Runges, welcher selbst mit Rücksicht auf die Spontan-

1) Meine Experimente waren bereits vor Palm's Veröffentlichung abgeschlossen. 
552 Blumreich, Experinente z. Frage nach d. Ursachen d. Geburtseintrittes.

contractionen gravide Kaninchen von seinen Experimenten ausschloss, seine Beobachtungen auch auf trächtige und geborenhabende Kaninchen mit ausdehnte und zwar unter Zuhülfenahme des Wasserbades. Auch Palm kam wie ich zu dem Resultat, dass „der vor dem Einfluss der Luft geschützte Uterus auch bereits geborenhabender Thiere, ja selbst der grapide Fruchtsack in verschiedenen Stadien der Trächtigkeit spontane Bewegserseheinungen erheblicher Art nicht zeigte, sodass er für die Versuche in gleicher Weise wie der jungfräuliche verwandt werden komnte."

Noch einige weitere Fehlerquellen müssen sorgfältig berücksichtigt werden, wenn man vor Täuschungen sicher sein will. Lässt man auf den in Ruhe befindlichen Kaninchenuterus einen Reiz einwirken, der energische Zusammenziehungen auslöst, so schliesst sich alsbald eine Summe von periodisch sich wiederholenden Contractionen an. Kehrer hat zuerst dieses Gesetz der rhythmischen Nachwirkungen aufgestellt. Man muss also, um neue Reize in ihrer Einwirkung auf die Wehenthätigkeit studiren zu können, erst das völlige Abklingen der rhythmischen secundären Uterus-Bewegungen nach der ersten Reizung abwarten.

Um die selbstständigen Bewegungen und Zuckungen des Thieres, durch welche dann wieder secundär rein mechanisch Uterus-Contractionen ausgelöst werden können, völlig fern zu halten, habe ich in einigen Vorversuchen mit Chloroform und Chloralhydrat gearbeitet. Das Chloroformiren der Kaninchen ist aber, wenn man die Thiere nicht verlieren will, sehr schwierig; zudem setzt es ebenso wie Chloralhydrat entschieden dio Wehenthätigkeit des Uterus in erheblichem Maasse herab. Ich habe daher sehr bald von diesen beiden Mitteln Abstand genommen und in der Mehrzahl der Fälle versucht, ohne Nareoticum auszukommen. Vergleichsweise habe ich in einer Reihe von Fällen Curare verwandt. Ich kann mich der Warnung Röhrig's und Runge's, grössere Dosen davon zu verabfolgen, nur anschliessen. Man sieht, dass entschieden die Erregbarkeit der Gebärmutter dadurch wesentlich leidet. Wie die beiden Autoren habe auch ich bei Verwendung kleinerer Quanten diesen Uebelstand, der zu einer wichtigen Fehlerquelle werden kann, nicht beobachtet.

Ein weiteres Moment, welches genaue Beobachtungen erfordert, ist die Ermüdung, welche der Uterus nach energiseher erfolgreicher Reizung häufig zeigt. Es kommt vor, dass z. B. nach Einwirkung eines Kohlensäuregemisches rom Blut aus der Uterus zunächst leb- 
hafte Contractionen zeigt, dass es aber bei weiterer Anwendung desselben Gasgemisches absolut nicht gelingt, eine Wiederholung der Zusammenziehung zu erzielen. Röhrig hat namentlich auf diese "Erschöpfung der Centren" aufmerksam gemacht, und empfohlen, zur Gewinnung deutlicher Resultate nur relativ schwache Reize zu verwenden und zwischen den einzelnen Reizen den Ganglien genügende Zeit zur Erholung zu gönnen. Ich möchte hervorheben, dass die verschiedenen Reizqualitäten sich hier verschieden verhalten, die Erschöpfung des Uterus ist nicht sowohl von der Reizstärke, als vielmebr von der Reizart abhängig; so tritt z. B. mechanischen, sehr intensiven Reizeinflüssen gegenüber nur sehr spät eine geringgradige Ermüdung auf, während gewisse chemische Reize bei geringerem Reizeffect als Erschöpfung der Uteruscentren bewirken.

Hierbei kommt meines Erachtens gewiss zum Theil in Betracht, dass manche Reizmittel, namentlich chemischer Natur, sehr schnell im Anschluss an die primäre Reizwirkung eine Lähmung zustandekommen lassen, wie das ja die Thatsachen der allgemeinen Muskelphysiologie nahe legen.

$\mathrm{Zu}$ den nothwendigen Cautelen gehört schliesslich noch eine äusserst behutsame Eröffnung der Leibeshöhle, nach einem langen Schnitt genau in der Medianlinie vom Proc. xiphoideus bis zur Symphyse werden die Wundränder vorsichtig mit Häkchen auseinandergezogen, die Blase, falls sie gefüllt ist und die Betrachtung stört, nach abwärts, die Därme, falls sie hinderlich sind, ganz zart nach oben umgelegt. Der Uterus selbst darf unter keinen Umständen dabei angefasst oder gar gezerrt werden.

Bei der Anstellung der Versuche unter den ausgeführten Cautelen war zunächst zu erwägen, ob man sich zur Constatirung der Reizwirkungen der graphischen Methode bedienen sollte oder nicht. Es ist ja fraglos, dass die Resultate ein wesentlich objectiveres Gepräge erhalten würden, wenn man eine Methode zur Verfügung hätte, die die Uterusbewegungen nach Reizung der Gebärmutter selbstthätig registrirte. Der Mangel einer solchen ist wohl von allen früberen Autoren sehr schmerzlich emptunden worden, ohne dass indessen die zahlreichen Arbeiten der vielen, zum Theil sehr angeseheneu Experimentatoren, eine brauchbare Methode gezeitigt hätten. Die Schwierigkeit liegt eben darin, dass der Apparat, der die Uterusbewegungen auf die Trommel übertragen soll, mit der Gebärmutter in sehr innigem Connex stehen muss; dadurch 
154 Blumreich, Experimente z. Frage nach d. Ursachen d. Geburtseintrittes.

werden aber sehr leicht mechanische Reize gesetzt, sodass man nun bei artificiellen Erregungen nicht mehr die Grenze zwischen Effekten durch die graphische Methode an sich und solchen durch die Reizung finden kann. Wie ich weiter oben ausführte, leidet auch die Methode Frommel's an diesem Uebelstand. Ich nahm daher von vornherein für meine Experimente von ihrer Verwendung Abstand. Ich habe dann noch auf verschiedene Weise versucht, eine graphische Methode ausfindig zu machen, bei der die mechanische Schädigung des Versuchsobjects eine möglichst geringe wäre. Indessen ist es mir trotz mühseliger Experimente nicht gelungen, hier zu einigermaassen befriedigenden Resultaten zu gelangen; stets wurde der Nutzen der directen graphischen Uebertragung durch den Schaden der mechanischen Reizung aufgewogen. Ich war daher auch wie die früheren Autoren gezwungen, bei der experimentellen Prüfung der Reize die Effekte durch das blosse Auge festzustellen. $\mathrm{Um}$ indessen hier nach Möglichkeit vor den Selbsttäuschungen, die einer subjectiven Methode ja nun einmal anhaften, gesichert zu sein, habe ich in sehr zahlreichen Versuchen Herrn Prof. Zuntz gebeten, mit mir die Beeiflussung des Uterus durch die angewandten Reize zu beobachten. Wir haben häufig das Protokoll gemeinschaftlich aufgenommen, häufig unabhängig von einander die gemachten Wahrnehmungen niedergeschrieben: der Vergleich ergab fast stets eine übereinstimmende Auffassung des Ergebnisses.

Dem subjectiven Ermessen bei der Beurtheilung der Resultate der Reizung wird ferner dadurch eine gewisse Grenze gesetzt, dass man verschiedene Arten von Contractionen, denen verschiedene Grade entsprechen, am Uterus des Kaninchens ohne Weiteres deutlich unterscheiden kann. Kehrer hat als Erster in seiner grundlegenden Arbeit über "die Zusammenziebung des weiblichen Genitalcanals" drei Formen von Zusammenziehungen am Fruchthalter des Kaninchens unterschieden. 1. Die stationäre Einschnürung oder die Strictur. 2. Die fortschreitende Zusammenziehung oder die Progressivcontraction. 3. Den Starrkrampf oder den Tetanus.

Als Reizeffekt schwächerer Natur kann man die stationäre Linschnürung auffassen. Die Strictur schliesst sich namentlich an mehr locale Erregungen an. Intensiver ist die Wirkung schon, wenn es sich um eine peristaltisch turtpflanzende Progressivcontraction handelt. Diese geordneten peristaltischen Bewegungen durchlaufen für gewöhnlich vom abdominellen Tubenende anfangend den Uterus bis herab zum Muttermund. Relativ seltener treten 
Blumreich, Experimente z. Frage nach d. Ursachen d. Geburtseintrittes. 155

antiperistaltische Contractionen auf, vom Muttermund aus nach oben ausstrahlend. Hier handelt es sich also um Contractionen allgemeiner Natur, die fortschreitend immer weitere Abschnitte des Uterus ergreifen. Am stärksten tritt der Einfluss der Erregung. hervor bei der tetanischen Zusammenziehung der Gebärmutter, hier richtet sich der Uterus sozusagen auf, drängt sich unter Aufbäumen nach vorn. Die Strictur kann bei Anwachsen der Reizung übergehen in die Progressivcontraction, ja sogar in den Tetanus uteri und ebenso sehen wir nicht selten, wie die peristaltische Welle, die den Uterus durchläuft, schliesslich in einer tetanischen krampfhaften Zusammenziehung endigt.

Ich habe geglaubt, der Besprechung der Methodik einen breiten Raum gewähren zu müssen, weil die Versuche ohne exacte Kenntniss und Beachtung der zahlreichen Fehlerquellen zu den verwirrendsten Schlüssen führen.

Da es mir darauf ankam, zu sehen, wie diejenigen Reize, welche für das Eintreten der Geburt bisher verantwortlich gemacht worden sind, den tragenden und den nicht schwangeren Uterus beeinflussen, speciell ob Unterschiede in dem Verhalten des Organs, je nachdem Gravidität vorliegt oder nicht, diesen Reizen gegenüber festzustellen sind, so schied von vornherein bei der Prüfung eine Reihe von Erregungsmitteln aus, die mit dem Beginn der Wehenthätigkeit sicher nicht in Beziehung gebracht werden konnten, wie elektrische und thermische Reize und viele toxische Substanzen. Dagegen erschien es mir in hohem Grade wünschenswerth zu sein, zu prüfen, wie eine experimentell erzeugte Kohlensäureüberladung des Blutes, ferner Sauerstoffmangel im Gefässsystem und schliesslich mechanische Reizungen auf den tragenden und nichttragenden Uterus einwirken, da einerseits erhöhte Venosität des Blutes sub finem graviditatis, andererseits steigender Druck auf die Cervicalganglien, also ein mechanisches Moment, zur Erklärung des Geburtseintrittes besonders herangezogen worden sind.

\section{Versuche mit Kohlensäureüberladung des Blutes.}

Die ersten Versuche, durch Kohlensäureüberladung des Blutes Uteruscontractionen auszulösen, rübren von Brown-Séquard her. Brown-Séquard operirte an zwei trächtigen Kaninchen. Dem ersten spritzte er in die Aorta kohlensäurereiches Hundeblut, in die Aorta des zweitens Kaninchens injicirte er kohlensäureärmeres von demselben Thier. Bei beiden hochtragenden Kaninchen kam 
Io6 Blumreich, Experimente z. Frage nach d. Lisachen d. Geburtseintrittes.

es alsbald zur Geburt, beim ersten 3 Minuten, beim zweiten 10 Minuten nach der Injection.

Auf dieses Experiment hin stellte Brown-Séquard die These anf, dass die Uteruscontractionen sub partu grösstentheils durch die Ueberladung des Blutes mit Kohlensäure zu erklären seien. Die Injection yon mehr oder weniger grossen Flüssigkeitsquanten in die Aorta setzt indessen an sich schon sehr lebhafte Uteruscontractionen, wie Kehrer gezeigt bat, und damit fällt die experimentelle Stütze der Ansicht B.-S.'s. Ich möchte hinzufügen, dass nach den Cntersuchungen von Landois, Naunyn, Ehrlich u.s. w. sich bei Injection des Blutes einer Thierspecies in das Gefässsystem einer anderen mancherlei Reizwirkungen erwarten lassen, die keineswegs nur auf den Kohlensäuregehalt zurückzuführen sind.

Nachdem schon Kehrer darauf hingowiesen hatte, dass der Tod durch Asphyxie ein bewegungsauslösendes Reizmoment für den Lterus bilde, stellten Oser und Schlesinger sehr umfassende Untersuchungsreihen an. Sie zeigten, dass nach "Athmungssuspension", d. h. nach Unterbrechung der am curarisirten Thier vorgenommenen künstlichen Respiration in wenigen Secunden die arterielle Röthe im Uterus in eine venöse überging, dass nach 10-30 Stunden dann der Uterus des - jungfräulichen, aber geschlechtsreifen - Kaninchens von einer allmählich zum Tetanus anschwellenden Progressivcontraction ergriffen wurde. Oser und Schlesinger suchen die Uterusbewegungen nach Athmungssuspension durch einen Reizungszustand im Gehirn in Folge der aufgehobenen Sauerstoffzufuhr zu erklären. Auf welchem Umwege nun auch die Contractionen zu Stande gekommen sind, jedenfalls zeigten die beiden Autoren, dass derartig hochgradig "dyspnoisches Arterienblut", wie es durch Athmungssuspension erzeugt wird, am jungfräulichen geschlechtsreifen Kaninchenuterus sehr lebhafte Zusammenziehungen auslöst.

Dass von Basch und Hoffmann nur in 5 unter 11 Fällen devitiche Uteruscontractionen nach Unterbrechung der künstlichen Athmung eintreten sahen, beruht wohl darani, dass sie an Hunden experimentirten, welche, wie in der Methodik besprochen, ja einen besonders wenig reizbaren Uterus besitzen. Denn wie Oser und Schlesinger betonen auch Cyon und namentlich Röhrig das regelmässige und rasche Eintreten von starken Bewegungen am Kaninchenuterus nach Athmungssuspension. Röhrig zeigte durch selir sinnreiche Experimente, dass dieser. Effect indessen gebunden 
ist an das Vorhandensein des Lumbalmarks. Wurde nämlich das Rückenmark vom letzten Brustwirbel nach abwärts mittelst glühenden Drahts vollkommen zerstört, so blieb die vorher prompte Reaction des Uterus auf erhöhte Venosität des Blutes stets aus bei Erhaltenbleiben der directen elektrischen Reizbarkeit der Gebärmutter. Wie es scheint, hat auch Röhrig diese Versuche nur am jungfränlichen Uterus ausgeführt, wenigstens finden sich weder im Text noch in den Protokollen Anhaltspunkte dafür, dass auch träehtige Individuen verwandt wurden.

Nicht so einheitlich wie die Wirkung der Atmungs-Suspension ist der Effect der "Aortencompression" beurtheilt worden. Nachdem Spiege]berg als erster auf die danach auftetenden BewegungsErscheinungen im Genitalapparat aufmerksam gemacht hatte, betonte Kehrer, dass nach Abklemmung der Aorta keine oder nur sehr schwache Contractionen in den rubenden Genitalien sich einstellten, was von Basch und Hoffmann bei 2 Versuchen an Hündinnen bestätigen konnten. Dagegen hoben dann wieder übereinstimmend Oser und Schlesinger, Körner und Röhrig in zahlreichen und exacten Untersuchungen die hohe Wirksamkeit der Aorten-Compression als contractionserregendes Mittel hervor.

Auch Tödtung dureh Verblutung, erzeugt sehliesslich nach Kehrer, Oser und Schlesinger. Röhrig starke Zusammenziehungen des Gebärorganes.

Zur selben Zeit wie Röhrig publicirte Runge sehr schöne Experimente, in denen er der Frage näher trat, ob bei der Wirkung des dyspnoischen Arterienblutes, wie dies namentlich in den Arbeiten von Oser und Schlesinger genau studirt worden war, die Herabsetzung des Sauerstoffgehalts oder die behinderte Kohlensäureansscheidung das wesentliche Princip wäre. Er suchte zunächst festzustellen, ob durch rasche Ueberladung des Blutes mit Kohlensäure, obne dass gleichzeitig Sauerstoffmangel eintrat, Uteruscontractionen ausgelöst werden. Zu diesem Zweck wurde das Thier tracheotomirt und eine Kohlensäure-Ueberladung des Blutes durch Inhalation in der Weise erzeugt, dass aus einem Waldenburg'schen Pneumanometer, welcher ein Gemenge von 1 Volum Sauerstoff und 2 Volum Kohlensäure enthielt, das Gasgemisch auf dem Umweg. über eine als Druckregulator wirkende Blase zu einer in die Trachea eingebundenen T-Canüle geleitet wurde. Gasanalysen ergaben, dass bei diesen Versuchen eine alleinige Vermehrung der Kohlensäurespannung erzielt wurde, ohne gleichzeitigen Sauerstoffmangel. 
$1 \breve{8} 8$ Blumreich, Experimente z. Frage nach d. Ursachen d. Geburtsointrittes.

"Diese Versuche ergaben nun übereinstimmend, dass in der That 30-40 Sekunden nach dem Beginn der Gasathmung ProgressivContractionen des Uterus eintreten, die sich fast stets zum wirklichen Tetanus steigern. Dieser ist aber nur von kurzer Dauer; es folgen noch einige Progressiveontractionen, dann wird der Uterus schlaff. Bei weiterer Kohlensäureathmung tritt dann keine Bewegung des Tragsackes mehr auf. Lässt man die Thiere indessen wieder rasch athmosphärische Luft athmen, so schwinden die Zeichen der Kohlensäureintoxication rasch und nach völliger Ruhe desselben hat dann die Kohlensäure-Inhalation wieder den gleichen Effect, lebhafte Bewegungen, selbst Tetanus der Gebärmutter".

Runge fasst das Ergebniss dieser Versuche dahin zusammen, dass die Kohlensäure Progressiv-Contractionen und tetanische $\mathrm{Zu}$ sammenziehungen erzeugt, aber nur in den ersten Minuten der Inhalation. In den tieferen Graden der Intoxication überwiege der Erschlaffungszustand. Auch sei die Intensität der Bewegungen nach Kohlensäureinbalation nicht immer die gleiche bei allen Versuchsthieren. Die Versuche, die Runge zu diesem Schluss führten, wurden sämmtlich am jungfräulichen Thier ausgeführt.

Mit den citirten Arbeiten von Spiegelberg, Cyon, Körner, Oser and Schlesinger, Röhrig und Runge, von denen namentlich die drei letzten auf die regelmässige und energische Wirkung des dyspnoischen Arterien-Blutes beim nichtschwangeren Thier aufmerksam gemacht hatten, steht die Mittheilung Cohnsteins in starkem Widerspruch. Cohnstein giebt an, dass AthmungsSuspension, Abklemmung der Aorta, Tod durch Verblutung in der Regel nur bei trächtigen Thieren, bei diesen aber constant, die Contractionen verstärke, dass dagegen die Musculatur des nichttragenden Uterus keine constante Reaction auf Veränderungen im Gasaustausch des Blutes zeige.

Die Ergebnisse, welche Cohnstein bei der Application dieser Reize hatte, sind in den eben angeführten Sätzen niedergelegt. Genauere Mittheilungen fehlen gänzlich, desgleichen die Angaben über die verwandte Thierzahl. Auch ist die Versuchsanordnung nur fragmentarisch wiedergegeben. Es ist daher die Beurtheilung seiner nicht im Einklang mit denen der übrigen Autoren stehenden Resultate kaum möglich.

Nach dem Vorgange von Runge suchte ich nun ebenfalls durch Inhalation eine Kohlensäure-Ueberladung des Blutes ohne Sauerstoffmangel herzustellen. Diese Experimente wurden unter 
den oben ausführlich besprochenen Cautelen an insgesammt 38 theils tragenden, theils nichttragenden Thieren vorgenommen und zwar in zwei Versuchsserien.

In der ersten Versuchsreihe wurde das Thier aufgebunden, tracheotomirt und in die Trachea eine T-Canüle eingebunden. Das eine Ende derselben blieb in freier Verbindung mit der atmosphärischen Luft, das andere wurde durch einen Schlauch mit einer Kohlensäurebombe verbunden. Durch Oeffnen des Hahns derselben bis zu einer ganz bestimmten Weite mischte sich dann das Gas mit der Inspirationsluft des Thieres und dieses Gemisch wurde durch Analyse nach Hempel's Methode auf seinen Gehalt an Kohlensäure und Sauerstoff untersucht. Es wurde darauf geachtet, dass die Verarmung an Sauerstoff niemals so hochgradig wurde, um an sich zur Dyspnoe Anlass zu geben. Die Kohlensäuremenge schwankte zwischen 40 und 60 Volum-Procent. Soll die Kohlensäureathmung unterbrochen werden, so wird die Kohlensäurebombe zugedreht.

Je nach der Stärke des Kohlensäurestromes und der Dauer seiner Anwendung tritt im Gesammtzustand des Thieres eine verschiedene Wirkung hervor. Der „Kohlensäurereizung" entspricht eine dyspnoische Athmung, die nach der Stärke des Stromes mehr oder weniger vertieft und frequent ist, dabei ist der Cornealreflex erhalten. Im weiteren Stadium der "Kohlensäurenarkose" wird die Athmung stossweise und viel weniger ausgiebig und die Cornea völlig unempfindlich.

Es zeigte sich nun, dass bei den meisten der nicht schwangeren Thiere der von Oser und Schlesinger, sowie Runge beschriebene Effect eintritt. Einige Secunden nach Einwirkung des Kohlensäurestromes machten sich leichte Bewegungen in der bis dahin ganz ruhig daliegenden Gebärmutter bemerkbar. Nach ungefähr 20-30 Secunden steigerten sich diese ganz leichten $\mathrm{Zu}$ sammenziehungen zu wirklicher Peristaltik des ganzen Organs, und in den meisten Fällen schloss sich ein Tetanus uteri an. Die Hörner des Uterus bäumten sich auf and wanden sich förmlich zu einem Knäul zusammen. Dieser Tetanus hielt indessen nur einige Secunden an, dann gingen die starren Zusammenziehungen über in peristaltische Wellenbewegungen, nach einigen weiteren Secunden waren auch diese abgeklungen. Bei manchen der Thiere konnte durch eine Verstärkung des Kohlensäurestromes bis zur Kohlensäurenarkose eine nochmalige energische Zusammenziehung der 
160 Blumreich, Experimente z. Frage nach d. Ursachen d. Geburtseintrittes.

Gebärmutter ansgelöst werden, andere blieben aber weiterhin völlig reactionslos. Wurde die Kohlensäureinhalation unterbrochen und atmosphärische Luft zugeführt, so gelang es in den meisten Fällen nach ca. 10 Minuten mit erneutem Kohlensäurestrom wieder Contractionen hervorzurufen. Bei einigen Thieren indessen war nach der einmal gelungenen Kohlensäurereizung jede Möglichkeit, das Organ wieder durch Kohlensäure zur Contraction zu bringen, geschwunden, wenigstens innerhalb der meist über 2 Stunden ausgedehnten Beobachtungszeit. Befanden sich die Thiere noeh nicht in geschlechtsreifem Alter, was man an der gelblichen Färbung der Gebärmutter und der Fadenform der Hörner ganz gut erkennen kann, so war die Reizung durch den Kohlensäurestrom meist sehr wenig wirksam, kaum, dass gelegentlich eine oder mehrere peristaltische Bewegungen eintraten; zum wirklichen Tetanus kam es nie.

Ganz anders verhielten sich nun die in derselben Weise dem Kohlensäurestrom ausgesetzten tragenden Kaninchen. Während bei dem nicht schwangeren Thier doch immer ein sehr deutlicher Iffect der Kohlensäureeinathmung, wemn auch in versehiedener Stärke, zu verzeichnen war, fehlte bei einigen der trächtigen Kaninchen eine jede Wirkung; selbst wenn die Kohlensäureinhalation bis zur tiefsten Narkose und bis nahe zum Tode fortgesetzt wurde, waren in keinem Stadium derselben Bewegungen nachweisbar. In der Mehrzahl der Fälle traten indessen Contractionen auf, die aber verspätet eintraten und dann an Stärke und Dauer weit hinter den am nichtschwangeren Thier beobachteten Bewegungen zurückblieber. Eine tetanische Zusammenziehung liess sich mit dieser Reizmethode am sehwangeren Thier überhaupt nicht erzielen; was zur Erscheinung kam, das waren locale Zusammenziehungen und höchstens Progressiveontractionen, die aber nach kurzer Zeit völlig aufhörten, durchschnittlich - mit der Secundenwhr gemessen - noch nicht halb so lange andauerten, wie bei der anderen Thiergruppe. Zur Geburt ist es bei keinem einzigen der verwandten hochtragenden Thiere im Verlauf des Versuchs gekommen.

Es konnte also beim schwangeren Thier durch die in der erwähnten Weise ausgeführte Kohlensäureathmung eine ähnlich ergiebige Locomotion des Gebärorgans wie beim nichttragenden absolut nicht erreicht werden, mit anderen Worten: es hat die Irritabilität des Uterus gegenüber der erböhten Kohlensäureüberladung des Blutes in der Sehwangersehaft erheblich abgenommen. 
BI umreich, Experimente z. Frage nach d. Ursachen d. Geburtseintrittes. 161

Ob wir es hier mit einem Zustand zu thun haben, der erst allmälig in der Gravidität Platz greilt - wie es ja wohl a priori anzunehmen ist, - oder ob mit der Befruchtung, also von Anfang der Schwangerschaft an eine verminderte Erregbarkeit dem vermehrten Kohlensäuregehalt des Blutes gegenüber besteht, darüber habe ich in dieser Versuchsreihe nichts Näheres feststellen können. Zufällig befanden sich nur zwei der benutzten tragenden Kaninchen in der, ersten bez. zweiten Woche der Schwangerschaft, alle ander"en Thiere standen kurz vor dem Werfen. Bei diesen beiden 'Thieren war die Erregbarkeit des Uterus allerdings eine grössere als bei den hochschwangeren, àber die Zahl ist natürlich viel zu klein, als dass man irgend welche sicheren Schlüsse daraus abzuleiten berechtigt ist.

Ich lasse einige Versuchsbeispiele aus dieser Serie hier folgen:

Kräftige Zibbe, $3600 \mathrm{~g}$, nicht tragend, hat noch nicht geworfen. 1 Uhr 05 Min. - Sec. Tracheotomie, Kochsalzwasserbad, Laparotomie, Uterus rosenroth, bandförmig, absolut ruhig.

\begin{tabular}{|c|c|c|c|c|c|c|}
\hline & " & 10 & $n$ & - & $n$ & $\begin{array}{l}\text { Einleitung d. Kohlensäurestromes in d. üblichen } \\
\text { Stärke. }\end{array}$ \\
\hline & $n$ & 10 & $\because$ & 15 & $n$ & $\begin{array}{l}\text { Ganz leise Bewegungen scheinen über den } \\
\text { Uterus hinwegzufahren, hier und da momentane } \\
\text { Einschnürungen, alsbald sich wieder lösend. }\end{array}$ \\
\hline & $\eta$ & 10 & $" 1$ & 28 & $\eta$ & Am Uterus beginnt eine sehr \\
\hline & $n$ & 10 & $n$ & & $n$ & lebhafte Peristaltik, rasche \\
\hline & $n$ & 10 & $"$ & 33 & $"$ & $\begin{array}{l}\text { wellenförmige Bewegungen, } \\
\text { ziemlich continuirlich sich an- }\end{array}$ \\
\hline & $"$ & 10 & 7 & 40 & $n$ & einander anschliessend. Uterus- \\
\hline & $"$ & 10 & $n$ & 41 & $"$ & $\begin{array}{l}\text { hörner werden ganz blass, } \\
\text { bäumen sich auf, Tetanus uteri. }\end{array}$ \\
\hline 1 & $\eta$ & 10 & $n$ & 46 & $n$ & $\begin{array}{l}\text { Tetanus beginnt sich zu ver- } \\
\text { lieren. }\end{array}$ \\
\hline 1 & $n$ & 10 & $n$ & 50 & $n$ & $\begin{array}{l}\text { Tetanus hat vollkommen nach- } \\
\text { gelassen, Wellenbewegung im } \\
\text { Uterus. }\end{array}$ \\
\hline & & & $n$ & 52 & $n$ & te Peristaltik. \\
\hline 1 & $"$ & 11 & $"$ & - & $"$ & Is völlig ruhig. \\
\hline & $n$ & 11 & $"$ & 02 & $"$ & $\begin{array}{l}\text { Verstärkung des Kohlensäurestroms, Athmung } \\
\text { sofort stossweise, Uterus ruhig. }\end{array}$ \\
\hline & $n$ & 11 & $n$ & 10 & $\eta$ & Cormealreflex erloschen, Uterus rubig. \\
\hline & $n$ & 11 & $n$ & 13 & $"$ & $\begin{array}{l}\text { Uterus ruhig, Unterbrechung des Kohlensäure } \\
\text { stromes, Luftathmung. }\end{array}$ \\
\hline & $n$ & 12 & , & 一 & $n$ & Thier erholt sich allmählich. \\
\hline & $n$ & 20 & $n$ & - & $n$ & $\begin{array}{l}\text { Wiederbolung des Versuchs (mit demselbe } \\
\text { Erfolg). }\end{array}$ \\
\hline
\end{tabular}
schnittlich. 
162 Blumreiol, Experimente z. Frage nach d. Grsachen d. Geburtseintrites.

11 Uhr 32 Nin. - Sec. Tracheotomie, Kochsalzwasserbad, Laparotomie, Uterus zeigt ganz leichte Bewegungen an zwei der schwangeren und einer leeren Partie der Gebärmutter.

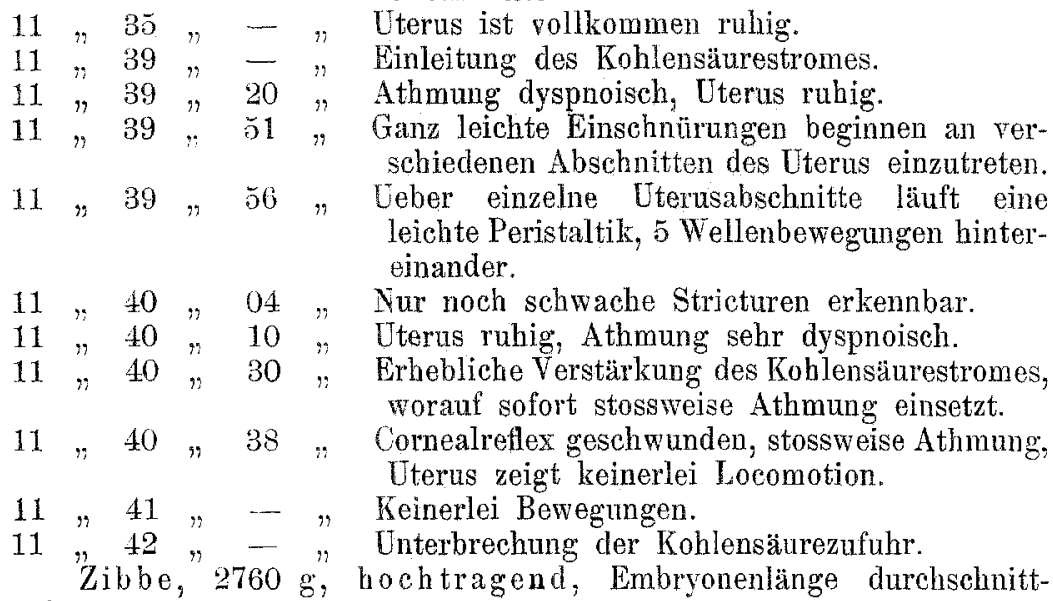
lich $9 \mathrm{~cm}$.

11 Uhr $22 \mathrm{Min}$. - Sec. Tracheotomie, Kocbsalzwasserbad, Laparotomie, Uterus zeigt keine Contractionen, Thier athmet rubig.

11 " 26 - " Beginn der Kohlensäureinhalation.

$11 " 26 " 06 "$ Athmung beginnt leicht dyspnoisch zu werden, Uterus ruhig.

11 "26 "20 * Atbmung stärker dyspuoisch, Uterus völlig bewegungslos.

$11 " 27 "$ - $"$ Uterus absolut ruhig.

$11 " 27 " 30 "$ "Uterus rubig. Verstärkung des Kohlensäurestromes.

11. "27 " 35 "Ganz leichte Zuckungen gehen durch einzelne Abschnitte des Gebärorgans.

$11 " 27 " 45 "$ Uterus schon wieder in Ruhe.

$11 " 27,55 "$ Uterus bleibt in Ruhe. Unterbrechung der Kohlensäureathmung.

$11 " 40 "$ - " Kohlensäureathmung mit demselben Erfolg.

Da in diesen Versuchen, obwohl die Zusammensetzung der Athemgase untersucht wurde, eine vorherige genaue Dosirung der Kohlensäurespannung unmöglich war, so versuchte ich in einer zweiten Versuchsreihe, den Bedingungen der Kunge'schen Versuchsanordnung möglichst nahe zu kommen, zumal es ja durchaus möglich war, dass die Verschiedenheit in der Reaction, welche dio einzelnen schwangeren, sowie die nichtschwangeren Thiere untereinander zeigten, auf quantitativen Differenzen im Kohlensäuregehalt des Blutes beruhten.

In der zweiten Versuchsreihe liess ich daher, wie Runge, 
ein Gasgemenge inhaliren, welches auf 2 Volumen Kohlensäure, ein Volum Sauerstoff enthielt.

In einem sorgfältig equilibrirten Quecksilberspirometer, nach Art der bei den Röhrig-Zuntz'schen Versuchen benutzten, wurde das zu athmende Gasgemisch aus zwei Theilen Kohlensäure und einem Theil Sauerstoff hergestellt. Das Spirometer iührte durch eine sich gabelnde Leitung zu zwei Müller'schen Wasserventilen, welche so angeordnet waren, dass das eine die Inspiration, das andere die Expiration ermöglichte, und pon hier aus zur Trachealkanüle des Thieres. Die Ventile waren bei diesen Versuchen mit Wasser gefüllt. In den Experimenten, welche am curarisirten Thier angestellt wurden, kam zur künstlichen Athmung die durch den Lehmann'schen Vacuummotor getriebene Quecksilberpumpe zur Anwendung, wie sie von Zuntz beschrieben und abgebildet worden ist.

Die Ergebnisse dieser Versuchsreihe entsprechen durchans den Erfahrungen, die ich bei der ersten Serie von Thieren gemacht hatte. Auch hier zeigte sich stets der starke Einfluss des erhöhten Kohlensäuregehaltes des Blutes auf das nichtschwangere Gebärorgan. Sobald man nicht an gar zu jungen, also geschlechtsreifen Thieren operirte, gerieth der Uterus regelmässig nach einigen Sekunden in mehr oder weniger stürmische peristaltische Bewegungen, die bei fortdauernder Athmung mit dem $\mathrm{CO}_{2} \mathrm{O}$-Gemisch in starre Zusammenziehungen des Tragsackes übergingen, um dann alsbald in den Ruhezustand zurückzukehren und in dieser Erschlaffung auch bei weiter fortgesetzter Kohlensäurerespiration zu verharren. Nur bei 2 jungfräulichen Thieren zeigte die Gebärmutter, ohne dass zwischendurch athmosphärische Luft inhalirt worden war, zweimal hintereinander das beschricbene sich schliesslich zu einem Tetanus uteri steigernde Spiel der Bewegungen. Dagegen war die Intensität und Extensität der Contractionen beim schwangeren Thier erheblich herabgesetzt. Man sah vielmehr locale Zusammenziehungen an manchen Abschnitten des Uterus als allgemeine Peristaltik oder gar Starrkrampf. Es fiel ferner auf, dass die eintretenden Bewegungserscheinungen sich überhaupt viel mehr an den leeren Uterusabschnitten ausprägten als an den durch die Eier ausgedehnten.

Besonders bemerkenswerth ist, dass in keinem einzigen der beobachteten Fälle die Inhalation der Kohlensäure von Abortus oder auch Partus praematurus gefolgt war. Zwei Thieren kamen 5 
164 Blumreich, Experimente z. Frage nach d. Ursachen d. Geburtseintrittes.

bez. 8 Stunden nach Abbruch des Versuchs nieder. Da die Thiere sich in der Zwischenzeit völlig erholt hatten, keinerlei Zeichen der Kohlensäureintoxication mehr boten und ausserdem die Embryonen in beiden Fällen ganz ausgetragen waren, dürfte es nicht bereehtigt sein, wollte man die in diesen Fällen zur rechten Zeit eingetretene Geburt als Effect der Kohlensäureathmung auffassen.

Der Typus der Bewegungen war genau der gleiche wie bei der ersten Serie, so dass ich mich auf ausführliche Schilderungen nicht einzulassen brauche und auf die dort gegebene Besprechung verweisen kann. Nur das eine möchte ich noch hervorheben, dass meine Vermuthung, die kleineren Unterschiede in der Stärke der Concentrationen, die zwischen den einzelnen Individuen in der Gruppe der schwangeren, sowie in der der nicht schwangeren Thiere hervorgetreten waren, seien auf ungleiche Dosirung der Kohlensäure in der Athmungsluft zu beziehen, sich als nicht richtig herausstellte. Es sind auch bei genau gleichmässigem Procentgehalt der Luft an Kohlensäure Differenzen bei den einzelnen Thieren der beiden Gruppen untereinander deutlich nachweisbar gewesen, so dass man also gezwungen ist, den Einflüssen der verschiedenen Individualität der Thiere einen ziemlich breiten Spielraum einzuräumen.

5 von den tragenden Thieren befanden sich bei der Ausführung der Experimente in der ersten Hälfte der Schwangerschaft. Der Uterus des einen derselben zeigte genau die gleich geringe Neigung zur Contraction unter Kohlensäureeinwirkung, wie das Organ beim hochtragenden Kaninchen, bei vier Thieren war die Erregbarkeit des Tragsackes eutschieden stärker als am Ende der Schwangerschaft, die Contractionen waren ergiebiger, traten schon früher auf, erreichten allerdings nie den stürmischen Charakter. welchen die peristaltischen Bewegungen beim nichttragenden Thiere meistens aufwiesen.

Von insgesammt 7, in den beiden ersten Wochen der Gravidität untersuchten Kaninchen zeigten also 6 deutlich stärkere Reaction des Uterusmuskels bei der Kohlensäureinhalation als die Thiere im hochtragenden Zustande. Es scheint mir nicht gewagt zu sein, daraus den Schluss zu ziehen, dass wir es mit einer gewissen Wahrscheinlichkeit hier mit einem Zustand zu thun haben, der sich erst allmähilich in der Gravidität ausbildet.

Fassen wir also die Resultate der beiden Versuchsreihen an insgesammt 16 nichttragenden und 22 tragen- 
den Kaninchen zusammen, so ergiebt sich, dass in der Empfindlichkeit des leeren und des trächtigen Uterus der Kohlensäureathmung gegenüber eine wesentliche Differenz besteht, dergestalt, dass die Reizbarkeit des Fruchthalters in der Schwangerschaft sehr erheblich nachlässt. Diese geringe Irritabilität spricht sich auch darin aus, dass es in keinem der Versuche gelungen ist, durch Kohlensäurerespiration den Eintritt der Geburt zu erzwingen.

Nach diesem Schlusssatz ist es nun interessant, einige Experimente von Runge, die nur nebenbei erwähnt werden, zu betrachten. Bei vier trächtigen Kaninchen, welche aber zu einem anderen Zweck dienten, wurde die Kohlensäureathmung in der üblichen Methode Runge's eingeleitet, ohne dass indessen die Bauchhöhle selbst geöffnet und die Genitalien einer Besichtigung unterzogen wurden. Es zeigte sich, dass bei 3 Thieren trotz stundenlanger Einwirkung der Kohlensäure auch nicht die Spur einer Geburtsthätigkeit eintrat. Bei dem vierten wurden 15 Minuten nach Einleitung der Kohlensäureathmung völlig reife Embryonen aussgestossen. Die Geburt dieser ausgetragenen Thiere wird aber von Runge nicht auf die Kohlensäureeinwirkung bezogen. „Die Jungen waren indessen völljg ausgetragen, so dass es sich ebenso gut um eine rechtzeitige Geburt handeln konnte."

Runge, der von dem Gesichtspunkt ausgeht, dass der Sauerstoffmangel im Blut einen intensiveren Reiz bedeutet als die Kohlensäureüberladung, führt, da er mit Rücksicht auf die Spontancontractionen am tragenden Thier nicht direct experimentirte, diese 4 Fälle an, um den etwajgen Einwand zu widerlegen, dass „vielleicht der tragende Fruchtsack eine grössere Irritabilität der Kohlensäure gegenüber besässe als der nicht schwangere". Mir scheint noch mehr aus ihnen hervorzugehen, nämlich der Hinweis darauf, dass im Gegentheil die Reizbarkeit in der Gravidität vielleicht abnimmt, da doch die Kohlensäure beim nichttragenden Thier entschieden ein Mittel zur Erzeugung kräftiger Wohen darstelite.

Es sind diese Fxperimente Runge's also ganz im Sinne meiner Versuche ausgefallen, nur dass $R$ unge, da er die Frage von einem anderen Gesichtswinkel aus ansah, naturgemäss garnicht erst in Erwägung zog, ob das negative Ergebniss bei seinen vier trächtigen Kaninchen nicht vielleicht auf einer verminderten Erregbarkeit beruhte. 
166 Blumreich, Experimente z. Frage nach d. Ursachen d. Geburtseintrittes.

Aehnlich liegen die Dinge bei den Experimenten Palms. Nachdem Herr Prof. Zuntz auf meine Veranlassung an Herrn Geheimrath Runge geschrieben hatte, dass meine Ergebnisse von den seinigen etwas abwichen, hat Palm die Versuche Runge's einer sorgfältigen Nachprüfung unterzogen - unter Ausschaltung einer möglicherweise ins Gewicht fallenden Fehlerquelle - dabei aber naturgemäss nur die Fragestellung zu Grunde gelegt, $o b$ in der That die Kohlensäureüberladung bez. der Sauerstoffmangel contractionsauslösend einwirkten; er kam wie Runge zu einem positivem Ergebniss. Auch als er die Experimente aut gravide Kaninchen ausdehnte, konnte er den bewegungsauslösenden Effect der beiden Reizmittel feststellen, da es ihm besonders darauf ankam, die Reizwirkungen überhaupt, sowie den graduellen Unterschied zwischen der Wirkung des Sauerstoffmangels im Blut einerseits, der Kohlensäureüberladung andererseits festzustellen, wird er auf Unterschiede im Verhalten der Thiere, je nachdem sie tragend oder nichttragend waren, nicht weiter geachtet haben - wenigstens erkläre ich es mir so, dass der Autor sich über diesen Punkt nicht weiter auslässt.

Es war nun zu erwägen, worauf diese verschiedene Reaction des tragenden und des leeren Uterus bei kohlensäurereicher Athmung zurückzuführen wäre. Es schien mir nicht undenkbar zu sein, dass diese Unterschiede auf Veränderungen des Stoffwechsels, die ja in der Schwangerschaft vermuthlich eintreten, beruhten. Mit Rücksicht darauf suchte ich, bevor ich die geschilderten Experimente weiter verfolgte, der Frage des Chemismus in der Gravidität näher zu treten. Als Frucht dieser Untersuchungen publicirte ieh vor zwei Jahren in diesem Archiv Untersuchungen über Blutalkalescenz (ausgeführt an 40, theils tragenden, theils nichttragenden Kaninchen und theils schwangeren, theils gesunden nicht schwangeren Frauen; bestimmt mittelst der Titrirmethode unter Anwendung des Löwy'schen Princips der Titrirung lackfarbenen Blutes). Es ergab sich, dass die Alkalescenzverhältnisse des Blutes durch die Schwangersehaft in constanter und bedeutender Weise beim Thier und Menschen abgeändert werden, und zwar steigt die Blutalkalescenz in dieser Zeit ganz beträchtlich, um im Wochenbett wieder abzusinken.

Es lag nahe, an die Möglichkeit zu denken, dass diese erhöhte Alkalescenz des Blutes durch Bindung eines Theiles der Kohlensäure die Wirkung derselben abschwäche. Als wahrscheinlich 
B l umreich, Experimente z. Frage nach d. Ursachen d. Geburtseintrittes. 167

möchte ich indessen diese Annahme doch nicht bezeichnen, denn bei der oft bis zur Narkose fortgesetzten Inhalation des kohlensäurereichen Gasgemisches muss es zur Neutralisation auch des überschüssigen Alkali und dann endlich $z u$ einer nahezu ebenso hohen Tension der freien Kohlenșäure, wie bei weniger alkalischem Blut kommen.

\section{Versuche mit Sauerstoffmangel des Blutes.}

Runge hatte in einsichtiger Weise erwogen, dass die contractionsauslösende Wirkung des dyspnoischen Arterienblutes in den Versuchen von $0 \mathrm{~s} \in \mathrm{r}$ und Schlesinger auf zwei Factoren beruhen könne, entweder auf der Herabsetzung des Sauerstoffgehalts oder auf der Zunahme der Kohlensäuremenge oder auf beiden zusammen. Nachdem er die oben geschilderte Wirksamkeit der raschen Ueberladung des Blutes mit Kohlensäure auf das nichttragende Kaninchen constatirt hatte, ging or dazu über, zu prüfen, wie der Fruchthalter sich bei Sauerstoffmangel ohne gleichzeitige Vermehrung der Kohlensäurespannung verhielte. Er leitete daher ebenfalls bei nichttragenden curarisirten Kaninchen künstliche Athmung mit chemisch reinem Wasserstoff ein.

Runge lässt sich über das Ergebniss dieser Experimente folgendermaassen aus: $\ldots$... so begannen schon nach wenigen Sekunden äusserst lebhafte Progressivcontractionen, welche sich rasch zum Tetanus steigern, ihm folgen neue Progressivcontractionen, dann wieder Tetanus, und dieser Wechsel dauert bis zum Tode des Thieres, ja der Starrkrampf des Uterus überdauert noch sekundenlang den Herzstillstand. Steuert man durch rechtzeitige künstliche Athmung von atmosphärischer Luft dem Tode, so erlöschen die Bewegungen des Uterus nicht sofort, sondern dauern auch jetzt. noch sekundenlang mit besonderer Lebhaftigkeit an - kurz die durch Sauerstoffmangel erzeugten Bewegungen des Uterus übertreffen . sowohl an Intensität wie Dauer bedeutend diejenigen, welche man im Beginn einer Kohlensäureinhalation erhält.

Ich habe nach dem Vorgang von Runge ebenfalls bei 6 nichttragenden Kaninchen die künstliche Athmung mit Wasserstoff durchgeführt, dann aber die Experimente anch auf 3 hochträchtige Thiere ausgedehnt. In das Quecksilber-Spirometer, welches in der vorigen Versuchsreihe das $\mathrm{CO}_{2} \mathrm{O}$-Gemenge enthielt, wurde aus einer Wasserstoffentwicklungsflasche der chemisch reine Wasserstoff eingeleitet. Die Ventile wurden bei diesen Versuchen zur Absorption der Kohlen- 
168 Blumreich, Experimente \%. Frage nach d. Ursachen d. Geburtseintrittes.

säure mit starker Kalilauge beschickt und ausserdem passirte die Inspirationsluft eine Flasche ammoniakalischer Kupferlösung zur Absorption des Sauerstoffes.

Ich kann nun Runge ganz bestätigen, was die stärkere Reizwirkung des absoluten Sauerstoffmangels im Blut beim nichttragenden Thier anbelangt. In der That sieht man, dass das Gebärorgan dabei von ganz gewaltigen krampfhaften Bewegungen ergriffen wird und dass die tetanische Zusammenziehung den vorherrschenden Contractionsmodus darstellt. Wenn aber Runge schreibt, dass diese Bewegungen - gegenüber den Versuchsthieren bei Kohlensäureüberladung - - beim Sauerstoffmangel bei allen Experimenten stets in gleicher Stärke auftreten, so kann ich mich ihm nicht anschliessen; obwohl ich hier nur geschlechtsroife, aber jungfräuliche Thiere benutzte, also möglichst gleichmässige Versuchsbedingungen von Seiten des Thiermaterials hatte, fand ich in der Reaction der einzelnen Individuen nicht geringe Verschiedenheiten: besonders die tetanischen Zusammenziehungen schwankten nach Stärke, Dauer und Häufigkeit durchaus. Was die schwangeren Thiere anbelangt, so zeigte sich auch bei allen, dass der Sauerstoffmangel im Blut ein energischeres Reizmittel für den Uterusmuskel bildet als die Kohlensäurevermehrung, dagegen ist der Fruchthalter des geschwängerten Kaninchens auch diesem Erregungsmoment gegenüber weniger empfindlich, als der des nichttragenden Thieres.

Vor allem fällt ins Auge, dass es bei keinem der 8 hochtragenden Thiere bei Einwirkung des Sauerstoffmangels gelungen ist, die Geburt herbeizuführen, obwohl die Embryonen, wie sich bei der Section der Thiere ergab, entweder ganz oder nahezu ausgetragen waren.

Die peristaltischen Contractionen an der schwangeren Gebärmutter setzen später ein, kriechen viel langsamer fort als beim nichttragenden Kaninchen, sind zeitlich und örtlich weniger ausgedehnt. Tetanus uteri trat hier auch auf, wenn auch nicht bei allen schwangeren Thieren, und wo er sich fand, war er oft mehr rudimentär, ]öste sich rascher wieder in Wellenbewegungen auf und wiederholte sich nicht so oft. Die individuellen Einflüsse bei den geschwängerten Thieren waren noch auffallender als bei den nichttragenden; Kaninchen aus früherer Schwangerschaftsperiode hatte ich leider nicht zur Verfügung.

Es ist also der absolute Sauerstoffmangel im Blut 
ein Agens, das auf die Gebärmuttermuskulatur des tragenden, wie des ungeschwängerten Thieres energischer einwirkt als die Kohlensäureüberladung. Ein Mittel zur Geburtseinleitung aber stellt er ebensowenig vor wie die letztere, wie denn überhaupt der tragende Fruchtsack bei Einwirkung dieses Reizes viel schwächer reagirt als das Organ im nichtschwangeren Zustande.

Selbst wenn wir indessen nun die besprochene Theorie von Leopold und Hasse bezüglich der Einwirkung der venösen Hyperämie annehmen, würde dieselbe uns doch nur zu der Annahme berechtigen, dass eine Verarmung des Blutes an Sauerstoff bez. eine schlechtere Blutdurchströmung des Uterusgewebes gegen Ende der Schwangerschaft stattfindet; ein absoluter Sauerstoffmangel kommt ja sicher unter physiologischen Verhältnissen nicht vor.

Es war daher zu prüfen, ob auch starke Verminderung des Sauerstoffgehaltes ähnliche Effecte, wie absoluter Mangel nach sich zöge. Ich habe daher 4 schwangere Thiere ein Gemisch von 90 Volumprocent Wasserstoff und 10 Volumprocent Sauerstoff athmen lassen und es hat sich bei diesen vier Thieren übereinstimmend gezeigt, dass die Sauerstoffverarmung ein erheblich geringeres Reizmittel für den graviden Fruchtsack darstellt, als der vollkommene Sauerstoffmangel. Die danach beobachteten Contractionen waren durchgehends erheblich schwächer, traten später ein und hörten früher auf, als wenn das Thier unter ausschliessliche Wasserstoffathmung gesetzt wurde.

Es war mir leider nicht möglich, noch weitere derartige Versuche anzustellen. Weittragende Schlüsse lassen sich aus $4 \mathrm{EX}-$ perimenten natürlich nicht ziehen. Soviel glaube ich indessen wohl sagen zu dürfen, dass die Sauerstoff-Verminderung im Gefässsystem aller Wahrscheinlichkeit nach geringere Reizeffecte als der complete Sauerstoffmangel beim schwangeren Thier auslöst.

\section{Versuche mit mechanischer Reizung.}

Soweit ich die Literatur übersehe, ist der Uterus der Versuchsthiere mechanisch, also etwa durch Drücken, Kneifen, Stechen im Wesentlichen nur beeinflusst worden, um festzustellen, ob er auf directe Reizung überhaupt noch reagirt, z. B. nachdem gewisse nervöse centrale Partien zerstört oder wenigstens ihre Bahnen zum Uterus durchschnitten waren. Die meisten Autoren, wie z. B. 
170 Blumreich, Experimente z. Frage nach d. Ursachen d. Geburtseintrittes.

Röhrig, dem wir ja sonst sehr schöne Experimente verdanken, erwähren die directe mechanische Reizung des Uterus nur ganz nebenbei; dagegen sind, soweit mir bekannt, solche Versuche in systematischer Weise bisher noch nicht angestellt worden.

Das Missliche derartiger Experimente liegt darin, dass man die mechanischen Reize nicht in absolut bestimmt dosirter Stärke einwirken lassen kann; denn ein zweckentsprechender Apparat, an dem man die Kraft der mechanischen Wirkung direct ablesen kann, fehlt, und würde selbst ein solcher construirt, so wäre damit auch noch nicht sehr viel gewonnen, weil die mechanisch traumatischen Reizeinflüsse ja sehr verschiedener Natur sein können. Ich versuchte dieser Schwierigkeit Herr zu werden dadurch, dass ich einmal mehrere Arten der Reizung in Anwendung brachte, zweitens durch Verwendung einer entsprechend grösseren Thierzahl und schliesslich durch Gegenüberstellung der nichtschwangeren und der tragenden Thiere. Besonders die unmittelbare Beobachtung, dort des leeren, hier des geschwängerten Uterus nebeneinander bei Application derselben Reizqualität ermöglicht ja eine ziemlich gcnaue Erkennung etwaiger Unterschiede in der Empfindlichkeit der inactiven und der tragenden Gebärmutter.

Eine sehr zweekmässige Form der mechanischen Reizung scheint mir die leichte Berührung mit einem leinen Haarpinsel zu sein, denn man kommt hier, bei Anwendung desselben Pinsels überhaupt nicht über eine bestimmte sehr geringe Reizstärke hinaus.

Weiterhin habe ich Stösse auf den Uterus einwirken lassen und zwar in der Weise, dass unter mässigem Druck quer über die Vorderfläche des Uterus herübergefahren wurde. Es sollte dieser Hodus eine ungefähre Nachahmung soleher Gewalten, Stösse sein, die den Unterleib in breiter Ausdehnung truffen. Schliesslich habe ich die Uterushörner zwischen zwei Fingern mehr oder weniger stark gedrückt, dabei immer nichtsehwangere und schwangere Thiere gegenüberstellend, und, soweit man dies mit den Fingern in der Gewalt hat, mit genau dem gleichen Kraftmaass.

Die Beobachtungen wurden unter den üblichen Cautelen an 7 nichttragenden und 11 graviden Kaninchen angestellt.

Es zeigto sich nun, dass durchgehends die Empfindlichkeit des Gebärorgans den geschilderten mechanischen Reizeinflüssen gegenüber in der Schwangerschaft gesteigert ist. Es spricht sich dieser Unterschied zwischen dem Organ im tragenden und ruhigen nichttragenden Zustand schon deutlich aus, wenn man derartig zarte 
Reize einwirken lässt, wie sie die einfache. Berührung mit dem Haarpinsel darstellt. Beim nichttragenden Individuum löst das leise Betupfen mit dem Pinsel eine ringförmige Einschnürung an der betreffenden Stelle aus. Diese Strictur lässt aber alsbald wieder nach, und zwar ohne dass sich eine peristaltische Bewegung über das ganze Organ oder auch nur grössere Abschnitte desselben anschliesst, wenigstens zeigte sich bei 6 von den 7 Thieren nur dieser Effect. Dieselbe ganz zarte Betupfung erzeugte am hochschwangeren sofort eine ausgesprochene peristaltische Contraction. Dieselbe setzte sich, wenn auch nicht über das ganze Organ, so doch über mehrere leere und durch die Frucht ausgedehnte Uteruspartien fort und trat 3-4mal hintereinander auf; auf eine einfache Strictur beschränkten sich die Bewegungserscheinungen nie. Dass derartig geringgradige Reize nicht etwa Tetanus auslösten oder auch nur eine den ganzen Uterus umspannende Peristaltik, ist ganz natürlich.

Das Kneifen des Uterus zwischen zwei Fingern wurde vom schwangeren Organ stets mit erheblich energischerer Reaction, sehr viel länger andauernden, ergiebigeren stärkeren Contractionen beantwortet als rom nichttragenden, und zwar war es gleichgültig, ob man bei der graviden Gebärmutter die leeren oder die durch die Eier gefüllten Uterusabschnitte kniff. Indessen genügte diese Reizart in der nur mässigen Stärke nicht, um die Geburt herbeizuführen.

Aeusserst stürmische Contractionsphänomene wurden dagegen durch die Application von Stössen gegen den Tragsack ausgelöst. Schon der nichtschwangere Uterus gerieth in tetanischen Krampf, der einige Zeit anhielt, dann in peristaltische Bewegungen, die sich resch folgten, überging, um nach einigen Secunden von Neuem in Starrkrampf zu verfallen. Dieser zweite Tetanus dauerte aber nur kürzere Zeit an, die nun eintretenden peristaltischen Contractionen waren langsamer und liessen alsbald ganz nach. Das ganze Spiel der Bewegungen nach dem Stoss dauerte durchschnittlich $1 \frac{1}{2}$ bis 4 Minuten, also auch hier waren starke individuelle Verschiedenheiten vorhanden. Lässt man, bevor diese Form der Bewegungserscheinungen ganz vorüber ist, einen zweiten Stoss in derselben Weise und Stärke auf die Vorderfläche der Gebärmutter auftreffen, so kommt es alsbald zu einer Wiederholung des Tetanus und der Progressivcontraction. Wio mir scheint, tritt eine Ermüdung des 
172 Blumreich, Experimente 2. Frage nach d. Ursachen d. Geburtseintrittes.

Fruchthalters bei Anwendung directer mëchanischer Reize erst recht spät ein.

Beim Uterus gravidus löst ein derartiger energischer Stoss nun eine wahre Fluth von Contractionen aus. Tetanus und Progressivcontractionen wechseln in bunter Folge ab, besonders die peristaltischen Bewegungen überstürzen sich förmlich. Die Dauer der Bewegungsphänomene ist eine ganz erheblich längere als beim nichtschwangeren Thier; oft kommt der Uterus erst nach 12 bis 15 Minuten zur Berulkgung. Eine Ermüdung des Organs dem directen mechanischen Reizeinfluss gegenüber scheint beim schwangeren Tragsack, noch später als beim nichttragenden Organ, erst nach Einwirkung sehr zahlreicher Reize, aufzutreten. Wurde nach etwa 10 Minuten der Stoss wiederholt, so gewannen die Bewegungen von Neuem an Kraft und Ausdehnung. Häufiger als dreimal habe ich diese Stösse in den genannten Zeitabständen indessen nicht wiederholt.

Von den 11 graviden Thieren befanden sich 6, wie die Längenbestimmung der Embryonen ergeben hat, kurz vor dem Werfen. Bei 2 von diesen 6 Thieren kamen die starken Bewegungserscheinungen nach dem dritten energisch auftreffenden Stoss nicht mehr zur Ruhe, sondern führten nach weiteren 12 bez. 18 Minuten unter gleichbleibender Stärke der Zusammenziehungen zur Ausstossung mehrerer Früchte; das Gleiche war der Fall bei einem Thier mit Embryonen aus dem Anfang der vierten Woche; es kam hier nach $16^{1 / 2}$ Minuten zu einem Partus praematurus. Es hat sich also die Geburt hier direct an die mechanische Reizung angeschlossen.

Bei den nicht hochtragenden Thieren war mit einer Ausnahme aus der allerersten Schwangerschaftszeit, wo die mechanischen Reize garnicht anders wirkten, als beim nichttragenden Kaninchen, stets eine Steigerung der Empfänglichkeit gegenüber den Traumen zu verzeichnen, doch stand dieselbe entschieden im Allgemeinen hinter der Reizempfindlichkeit beim hochschwangeren Thier zurüek: nur ein Thier bildet, wie erwähnt, hier die Ausnahme; die mechanischen Reize wirkten so stark, dass es zur Frühgeburt kam.

Als Lrgebniss dieser Versuchsreihe kann ich sagen: Die auf verschiedene Art bewirkte directe mechanische Reizung bildet ein starkes Erregungsmittel für das Gebärorgan. Der mechanische Reiz wird indessen vom schwangeren Uterus mit sehr viel energischeren Contractionen beant- 
wortet, als vom nichtschwangeren Tragsack, ja es giebt mechanische Reizeinflüsse, die so lebhafte Bewegungen auslösen, dass sich unter weiter fortdauernden Contractionen die Geburt bez. Frühgeburt anschliesst.

\section{Schlussbetrachtung.}

Es hat sich also gezeigt, dass weder die acute Kohlensäureüberladung des Blutes, noch der absolute oder relative Sauerstoffmangel als ein Mittel zu betrachten ist, das beim Thier experimentell den Geburtseintritt herbeiführt, ja, dass sogar der Fruchthalter im tragenden Zustand eine deutliche Abnahme der Erregbarkeit beiden Agentien gegenüber aufwcist; ferner ergab sich, dass im Gegensatz dazu die Fähigkeit des Gebärorgans, auf Reize mechanischer Natur mit Bewegungserscheinungen zu reagiren, im Verlauf der Gravidität mit dem Weiterfortschreiten derselben erheblich wächst, ja, dass es geljngt, durch mechanische Einwirkungen mässig starker Natur solche Contractionen hervorzurufen, dass es schliesslich zur Ausstossung der Frucht kommt.

Es knüpfen sich hieran einige Erwägungen bezüglich der Theorien über die Ursachen des Geburtseintritts.

Die Theorie von Brown-Séquard, Leopold, Hasse von der venösen Hyperämie als auslösenden Factors der Wehenthätigkeit - eine Ansicht, auf deren, wie mir scheint, unzureichende anatomische Begründung ich in dem ersten Abschnitt dieser Arbeit eingegangen bin - hatte eine starke experimentelle Stütze in den Untersuchungen Oser's, Schlesinger's und Runge's gefunden, von denen die beiden ersteren festgestellt hatten, dass "dyspnoisches Arterienblut" ein energisches, contractionsauslösendes Mittel für den jungfräulichen Uterus bildet, während Runge weiter nachwies, dass zwar Kohlensäureüberladung sowohl als Sauerstoffmangel im Blut den Uterus des nicht graviden Thieres zu starken Bewegungen reizen, dem letzteren Moment indessen die energischere Wirksamkeit von beiden experimentell geprüften Factoren zukommt.

Diese experimentellen Pfeiler werden aber der genannten Theorie in dem Moment entzogen, wo nachgewiesen ist, dass Kohlensäureüberladung und Sauerstoffmangel des Blutes auf den tragenden Uterus schwächer einwirken, als auf den nicht schwangeren. Denn wären diese Factoren wirklich die geburtseinleitenden Momente, so müssten sie das schwangere Organ zum Mindesten ebenso 
174 Blumreich, Experimente z. Frage nach d. Ursachen d. Geburtseintrittes. stark erregen, wie die nicht tragende Gebärmutter - nicht aber, wie aus meinen Versuchen hervorgeht, erheblich schwächer.

Nun könnte man allerdings einwenden, ob überhaupt durch solche Untersuchungen, wie sie 0 ser und Schlesinger und Runge angestellt haben, etwas für oder gegen die Theorie bewiesen wird. Denn, so könnte man weiter sagen, ein derartig acut einsetzender und absoluter Sauerstoffmangel und eine so acute hohe Koblensäurespannung, wie sie hier experimentell geschaffen wurden, kommen sicher unter physiologischen Verhältnissen bei der Geburt nicht vor; alle Autoren, die für die Bedeutung der Blutgase als wehenauslösendes Mittel eingetreten sind, haben nur glaubhaft machen können, dass es sich hier um eine allmälig eintretende orhöhte Venosität des im Uterus circulirenden Blutes handele. Vielleicht wirken Sauerstoffmangel und Kohlensäure viel stärker auf den Cterus ein, wenn sie sich allmälig entwickeln und nicht so hochgradig sind?

Was den Sauerstoffmangel anbelangt, der ja nach Runge's und meinen Versuchen jedenfalls das wirksamere Reizmittel bildet, so zeigte sich in meinen vier nach dieser Richtung hin angestellten Lxperimenten, dass der tragende Fruchthalter bei der Inhalation sauerstoffarmer Luft erheblich schwächer reagirte, als bei sauerstofffreier. Also der - physiologisch nicht in Betracht kommende - absolute Sauerstoffmangel wirkt stärker auf den Tragsack ein, als der relative. Ferner haben ältere Versuche von Zuntz ergeben, dass bei ganz allmälig einsetzendem Sauerstoffmangel Bewusstlosigkeit und Tod des Thieres ohne vorausgegangene motorische Erregung zu Stande kommt. Das Gleiche lehren die Erscheinungen der langsamen Kohlenoxydvergiftung, die ja eigentlich auch nur fortschreitende Sauerstoffverarmung des Blutes ist; auch hier fehlen Reizerscheinungen von Seiten des motorischen Apparates.

Diese Beobachtungen sprechen nicht dalür, dass der relative und allmälig einsetzende Sauerstoffmangel ein energischeres Reizmittel als der absolute darstellt.

Was die Kohlensäureüberladung anbelangt, so ist es nicht sehr wahrscheirlich, dass eine allmälig sich entwickelnde geringgradigere Kohlensäureanhäufung im Blut auf den tragenden Uterus stärker wirkt, als auf den nicht tragenden, also den umgekehrten liffect haben sollte, wie die acute starke Kohlensäurc- 
Blumreich, Experimente z. Frage nach d. Ursachen d. Geburtseintrittes. 175

überladung, welche ja, wie gezeigt, beim nicht schwangeren Uterus lebhaftere Bewegungen auslöst, als beim sehwangeren. So lange also nicht durch exacte $V$ ersuche etwas Derartiges bewiesen ist, wird man dem Ausfall meiner Experimente gegenüber wohl sagen müssen: Kohlensäureüberladung und Sauerstoff mangel des Blutes wirken nicht derart auf den Uterus ein, dass sie als einleitende Factoren für den Geburtsact in Betracht kommen können.

Was schliesslich nun die klinische Seite der Frage betrifft, so braucht die Frühgeburt, welche man mitunter im Anschluss an incompensirte Herzfehler oder chronische Lungenerkrankungen auftreten. sieht, nicht nothwendig auf die Reizwirkung des stärker venösen Blutes zurückgeführt zu werden - wie das manche Autoren behauptet haben. Es könnte sich sehr wohl hier einfach um Einflüsse der in Folge der schlechteren Bluteirculation mangelhaften Ernährung, vielleicht auch um dyspnoische Bewegungen des Fötus und dadurch hervorgerufene mechanische Erregungen des Uterus handeln.

Dem negativen Resultate meiner Versuche mit Kohlensäureüberladung und Sauerstoffmangel am schwangeren Uterus stehen nun die positiven Ergebnisse mit mechanischer Reizung gegenüber. Mit dieser Beobachtung stimmt die klinische Erfahrung durchaus überein. Wir wissen, dass die schwangere menschliche Gebärmutter um so leichter durch mechanische Reize, Traumen irgend welcher Art zur Contraction gebracht wird, je weiter die Gravidität vorgesehritten ist; und diese Wahrnehmungen haben ja auch ihren Ausdruck gefunden in der allgemein verbreiteten Lehre der "erhöhten Irritabilität des Uterus in der Sehwangerschaft". Eine solche besteht allerdings, aber - und das ist ein wesentlicher Punkt meiner Ausführungen - sie ist keineswegs eine allen Reizen gegenüber in gleicher Weise ausgesprochene Erregbarkeit; denn es giebt eben Reizeinflüsse, wie Kohlensäureüberladung des Blutes und Saverstoffmangel, auf welche das Gebärorgan in der Schwangerschaft viel schlechter reagirt, als ausserhalb dieses Zustandes, während seine Empfänglichkeit für mechanische Erregungen deutlich zunimmt. Diese Ergebnisse werden noch ergänzt durch die in dem Abschnitt "Uebersicht über die bisherigen Reizversuche am Uterus" mitgetheilten Experimente verschiedener Autoren, aus welchen mit 
176 Blumreich, Experimente z. Frage nach d. Ursachen d. Goburtseinurittes.

grosser Wahrscheinlichkeit hervorgeht, dass die Empfindlichkeit des Fruchthalters auch elektrischen Reizen gegenüber in der Gravidität sinkt. Es handelt sich also beim Uterus um ähnliche Verhältnisse wie beim Gehirn; auch die Reizbarkeit der motorischen Hirncentren nimmt in der Scbwangerschaft zu, aber ebenfalls nicht allen, sondern nur bestimmten Medien gegenüber, wie ich in zum Theil gemeinsam mit Leo Zuntz angesteliten Versuchen zeigen konnte.

Bei der weiteren Forschung wird man diese Thatsache der ungleichmässigen Empfänglichkeit des graviden Uterus für die verschiedenen Reizeinwirkungen berücksichtigen müssen; denn es leuchtet ohne Weiteres ein, dass Factoren, die man als geburtseinleitende anspricht, sich bei der experimentellen Prüfung als besonders starke Erregungsmittel für den schwangeren Fruchthalter erweisen müssen.

Bei den mechanischen Reizen hat sich dieses Verhalten nun herausgestellt; und man ist daher mejnes Erachtens berechtigt zu sagen: Reizeinwirkungen mechanischer Natur können im Gegensatz zur Kohlensäureüberladung und zum Sauerstoffmangel des Blutes eine wesentliche Bedeutung für den Eintritt der Wehenthätigkeit besitzen. Es entspricht diese experimentell gewonnene Ansicht bezüglich der Wichtigkeit der mechanisch-traumatischen Momente auch der Auffassung Olshausen's, der in seinem Lehrbuch der Geburtshülfe schreibt: ...... dass gegen Ende der Schwangerschaft der Uterus immor mehr geneigt wird, auch auf zufällige äussere Ursachen hin, seien sie traumatischer (Olshausen fügt hinzu: oder psychischer) Natur zu reagiren, sodass der genauere Termin der Geburt doch häufig durch äussere Ursachen bedingt wird".

Mit diesem Nachweis der Bedeutung mechanischer Erregungen ist natürlich keineswegs erwiesen, dass das mechanische Moment stets der ausschlaggebende Factor beim Geburtseintritt ist; vielmehr könnten sehr wobl auch noch andere Reizwirkungen, die ja vorläufig noch hypothetischer Natur sind, hier mitspielen.

Will man die mechauischen Reize zur Erklärung des Geburtseintritts heranziehen, so müsste man besonders auch daran denken, dass ja mit fortschreitender Volumszunahme des Uterusinhaltes und fortschreitendem Wachsthum des Fötus die von diesem ausgehenden mechanischen Reize von Tag zu Tag stärker werden.

Mit dieser Thatsache und dem Nachweis, dass die Gebärmutter 
gewissen anderen Reizen gegenüber in der Schwangerschaft versagt, dagegen zunehmend empfänglicher wird für mechanische Erregungen, könnte dann der Geburtseintritt in vielen Fällen, wie mir scheint, ziemlich hinreichend erklärt werden. Schwierigkeiten macht allerdings immer der Umstand, dass der Endtermin ein so gesetzmässiger ist, dass er nur wenig durch stärkere Entwickelung und kräftigere Bewegungen des Fötus verfrübt wird, und es entzieht sich vorläufg unserer Kenntniss, welche Momente hier mitspielen, ob vielleicht ein Fortwirken der menstruellen Periodicität, wie das vielfach angenommen wurde, eine entscheidende Rolle spielt.

\section{Li t e r a t ur.}

1. v. Basch und Hoffmann, Untersuchungen über die Innervation des Uterus und seiner Gefässe. Wiener med. Jahrbücher, 1877.

2. Bumm, Dieses Archiv, Bd. 24.

3. Blumreich, Einfluss der Gravidität auf die Blutalkalescenz. Ein Beitrag zur Veränderung des Chemismus in der Schwangerschaft. Dieses Archiv, Bd. 59.

4. Blumreich und L. Zuntz, Experimentelle und kritische Beiträge zur Eklampsie. Dieses Archiv, Bd. 65.

5. Blumreich, Totale Urinretention beim schwangeren und nichtschwangeren Thier. Dieses Archiv, Bd. 66.

6. Brown-Séquard, Journ. de la physiol. de l'homme u. s. w. 1858.

7. Cyon, Pflüger's Archir, Bd. 8.

8. Calliburcès, Compt. rend. de l'acad. des sciences. 1857. p. 1095.

9. Cohnstein, Dieses Archiv, Bd. 18.

10. Conrad, Correspondenzblatt für Schweizer Aerzte. 1884. Citirt nach Werth in P. Müller's Handbuch der Geburtshülfe. Bd. I.

11. Dembo, De l'influence de l'électricité sur les contractions atérines. Soc. de biolog. 1883. p. 12.

12. Dohrn, Beitrag zur Anatomie der reifen menschlichen Eihüllen. Monatsschrift für Geburtskunde. Bd. 26.

13. Dohrn, Kritik der Arbeit von Friedländer. Dieses Archiv, Bd. 2.

14. Feis, Dieses Archiv, Bd. 46.

15. Frankenhäuser, Die Nerven der Gebärmutter. Jena, 1867.

16. Friedländer, Physiologisch-anatomische Untersuchungen über den Uterus. Leipzig, 1870.

17. Frommel, Zeitschrift für Geburtshülfe und Gynäkologie, Bd. 8 .

18. Geyl, Dieses Archir, Bd. 17. 1881.

19. Godet, Rech. sur la stricture intime du placenta du lapin. Diss. 1877. 
178 Blumreich, Experimente z. Frage nawh d. Usachen d. Geburtseintrites.

20. Hasse, Die Ursachen des rechtzeitigen Geburtseintritis beim Menschen. Zeitschr. 1. Gyn. und Gob. Bd. b.

21. Jakub, Ueber die rhytmischen Bewegungen des Kaninchenuterus. Arch. f. Anat. und Physiol. Physiol. Abth. 1884.

22. Jastreboff, Die Contractionen der Vagina beim Kaninchen. Arch. für Anat. und Physiol. Physiol. Abth. 1885.

23. Kehrer, Leber die Zusammenziehungen des weiblichen Genitalkanals. Beiträge zur vergleichenden und experimentellen Geburtskunde. Giessen, 1864. Heft 1.

24. Keilman, Zeitschr. für Gyn. und Geburtshülfe, Bd. 22.

25. Kilian, Einfluss der Med. obl. auf die Bewegung'en des Uterus. 'Zeitschr. für ratione]le Medicin. Nene Folge, Bd. 2.

26. Kilian, Ueber die Geburtslebre von Seiten der Wissenschaft und Kunst. Bd. 1. 1847.

26a.Knüpfer, Berichte und Arb. aus der Dorpater Frauenklinik. Wiesbaden, 1894.

27. Körner, Studien des physiologischen Instituts zu Breslau (Heidenhain). Heft 3 .

28. Kundrat und Engelmann, Untersuchungen über die Uterusschleimhaut. Wiener med. Jahrbücher, 1873.

29. Langhans, Zur Kenntniss d. menschlichen Placenta. Dieses Arch. Bd. 1.

30. J ehmann, Dubois Archiv f. Physiol. 1883.

31. Leopold, Dieses Arohiv, Bd. 11. S. 499.

32. Liedtke, Kritische Betrachtungen über die Ursachen u. s. w. Dissertation. Berlin, 1883.

33. Litzmann, De causa partum efficienti. Diss. 1843. Cit. nach Knäpfer.

34. Markwald, Ueber die Wirkung von Ergotin u. s. w. Arch. f. Anat. und Physiol. Physiol. Abth. 1883.

35. Sigmund Mayer, In Hermann's Handbuch der Physiologie.

36. Mauriceau. 'Trait. des malad. des femmes gross. etc. Livre 2. 1868. Citirt nach Kin üpfer.

37. Nägele, Versuch eines Studiums der Geburtshülfe. 1812.

38. Nikitin, In Rossbach's pbarmacentischen Untersuchungen. 1879. Bd. 3.

39. Obernior, Experimentelle Untersuchungen über die Nerven des Uterus. Bonn, 1865.

40. Olshausen-Veit, Lohrbuch der Geburtshülfe. 1899.

41. Oser und Schlesinger, Experimentelle Untersuchungen über Uterusbewegungen. Wiener med. Jahrbücher, 1872.

42. Palm, Monatsschr. für Geburtshülfe and Gyn. Bd. 14.

43. Petit, Mémoir sur le mechanisme et la cause de l'accouch. Recueil des pièces etc. 1766. Citirt nach Kn ̈̈pfer.

44. Reimann, Dieses Archiv, Bd. 2.

45. Ritgen, Die Triebfeder der Geburt. Gemeinsame Zeitschrift für Geburtskunde. Bd. 4.

46. Röhrig, Experimentelle Untersuchungen über die Physiologie der Cterusbewegungen. Virchow's Archiv, Bd. 76.

47. Röhrig-Zuntz, Pflüger's Archiv, Bd. 4. 
Blumreich, Experimente z. Erage nach d. Ursachon d. Geburtseintrittes. 179

48. Runge, Zeitschr. für Geburtshülfe und Gyn. Bd. 4.

49. Runge, Dieses Archiv, Bd. 13.

50. Schlesinger, Ueber Reflexbewegungen des Uterus. Wiener med. JahrJahrbücher, 1873.

51. Schlesinger, Ueber die Centren der Gefäss- und Uterusvenen. Wiener med. Jahrbücher; 1874.

52. Spiegelberg, Experimentelle Untersuchungen über die Nervencentren und die Bewegungen des Uterus. Zeitschr. für rationelle Medicin. Neue Folge, Bd. 2.

53. Spiegeiberg, Die Nerven und die Bewegungen der Gebärmutter. Monatsfür Geburtskunde, Bd. 24.

54. Veit, In Peter Müller's Handbuch der Geburtshülfe, Bd. 1.

55. Winkler, Zur Réenntniss der menschlichen Placenta. Dieses Arch. Bd. 4.

56. Zuntz, Pflüger's Archiv, Bd. 17. 